\title{
Taxonomic revision and molecular phylogenetics of the Idarnes incertus species-group (Hymenoptera, Agaonidae, Sycophaginae)
}

Fernando H A Farache ${ }^{\text {Corresp.. }}{ }^{1}$, Astrid Cruaud ${ }^{2}$, Gwenaëlle Genson ${ }^{2}$, Jean-Yves Rasplus ${ }^{2}$, Rodrigo A S Pereira

${ }^{1}$ Departamento de Biologia, FFCLRP, Universidade de São Paulo, Ribeirão Preto, SP, Brazil

2 Centre de Biologie pour la Gestion des Populations, INRA, Montferrier-sur-Lez, France

Corresponding Author: Fernando H A Farache

Email address: fhafarache@gmail.com

Sycophaginae is a group of non-pollinating fig wasps considered closely related to the fig pollinators (Agaoninae, Tetrapusiinae, and Kradibiinae) in the most recent phylogenetic analyses. They occur in all tropical regions and are associated with Ficus subgenera Urostigma and Sycomorus. There are six described genera of Sycophaginae, and two are native and confined to the Neotropics, namely Idarnes Walker, 1843 and Anidarnes Bouček, 1993. Genus Idarnes is divided into three morphologically distinct groups that were proven to be monophyletic by recent molecular phylogenetic analyses. In this paper we reviewed the Idarnes incertus species-group and provide detailed morphological descriptions and illustrations for the species belonging to this group. Three previously described species were redescribed: I. brasiliensis comb. nov. (Mayr, 1906) , I. hansoni Bouček, 1993, and I. incertus (Ashmead, 1900). Seventeen new species are described by Farache and Rasplus: I. amazonicus sp. n., I. amacayacuensis sp. n., I. americanae sp. n., I. badiovertex sp. n., I. brevis sp. n., l. brunneus sp. n., l. comptoni sp. n., I. cremersiae sp. n., I. dimorphicus sp. n., I. flavicrus sp. n., I. flaviventris sp. n., I. gibberosus sp. n., l. gordhi sp. n., I. maximus sp. n., I. nigriventris sp. n., I. pseudoflavus sp. $\mathbf{n}$. and I. ramirezi sp. $\mathbf{n}$. We provided keys for the identification of the species as well as for recognising the different species-groups of Idarnes and a closely related genus (Sycophaga Westwood, 1840). Additionally, phylogenetic relationships among $13 \mathrm{I}$. incertus species-group species were inferred using four molecular markers and discussed in the light of Ficus taxonomy and host specificity. 
urn:lsid:zoobank.org:pub:22286699-8306-4931-8D7F-7BF05EB2B304

Fernando Henrique Antoniolli Farache1, Astrid Cruaud ${ }^{2}$, Gwenaëlle Genson ${ }^{2}$, Jean-Yves Rasplus $^{2} \&$ Rodrigo Augusto Santinelo Pereira ${ }^{1}$

${ }^{1}$ Depto de Biologia/FFCLRP-USP, Av. Bandeirantes, 3900, 14040-901 - Ribeirão Preto, SP, Brazil; FHAF:fhafarache@gmail.com. RASP: raspereira@ffclrp.usp.br.

2 INRA-UMR Centre de Biologie pour la Gestion des Populations, CBGP, (INRA/ IRD/CIRAD/Montpellier SupAgro), Campus International de Baillarguet, CS 30016, 34988 Montferrier-sur Lez, $\quad$ France; $\mathrm{AC}$ : cruaud@supagro.inra.fr;

GG: gwenaelle.genson@supagro.inra.fr; JYR: rasplus@supagro.inra.fr.

--- Correspondence should be sent to: Fernando Henrique Antoniolli Farache Depto. de Biologia/FFCLRP, Universidade de São Paulo, Av. Bandeirantes 3900, Ribeirão Preto/SP, 14040-900, Brazil, Tel. +55 (16) 3315-4678, Fax. +55(16) 3315-4886, Email: fhafarache@gmail.com.

21

\section{Abstract}

Sycophaginae is a group of non-pollinating fig wasps considered closely related to the fig pollinators (Agaoninae, Tetrapusiinae, and Kradibiinae) in the most recent phylogenetic analyses. They occur in all tropical regions and are associated with Ficus subgenera Urostigma and Sycomorus. There are six described genera of Sycophaginae, and two are native and confined to the Neotropics, namely Idarnes Walker, 1843 and Anidarnes Bouček, 1993. Genus Idarnes is divided into three morphologically distinct groups that were proven to be monophyletic by recent molecular phylogenetic analyses. In this paper we reviewed the Idarnes incertus species-group and provide detailed morphological descriptions and illustrations for the species belonging to this group. Three previously described species were redescribed: I. brasiliensis comb. nov. (Mayr, 
32 1906), I. hansoni Bouček, 1993, and I. incertus (Ashmead, 1900). Seventeen new species are

33 described by Farache and Rasplus: I. amazonicus sp. n., I. amacayacuensis sp. n., I. americanae

34 sp. n., I. badiovertex sp. n., I. brevis sp. n., I. brunneus sp. n., I. comptoni sp. n., I. cremersiae 35 sp. n., I. dimorphicus sp. n., I. flavicrus sp. n., I. flaviventris sp. n., I. gibberosus sp. n., I. gordhi 36 sp. n., I. maximus sp. n., I. nigriventris sp. n., I. pseudoflavus sp. n. and I. ramirezi sp. n. We 37 provided keys for the identification of the species as well as for recognising the different species38 groups of Idarnes and a closely related genus (Sycophaga Westwood, 1840). Additionally, 39 phylogenetic relationships among 13 I. incertus species-group species were inferred using four 40 molecular markers and discussed in the light of Ficus taxonomy and host specificity.

41

42

43

44 45

46

47 48 49 50 51 52 53 54 55 56 57 58 59 60 61

\section{Introduction}

Fig trees (Ficus spp., Moraceae) host diverse assemblages of wasps that use the fig inflorescences (syconia or figs) to reproduce and develop. Fig pollinators (subfamilies Agaoninae, Tetrapusiinae, and Kradibiinae) form a very specialized clade of wasps that enter the fig trough a small pore enclosed by bracts, called ostiole. They lay eggs in the ovaries of pistilate flowers (Galil \& Eisikowitch 1969) and pollinate. Several other lineages of chalcid wasps use the fig to oviposit but do not pollinate, and are referred to as non-pollinating fig wasps (NPFW). These wasps exhibit variable life history traits (Elias et al. 2008; Pereira et al. 2007; Tzeng et al. 2008). They are gallers, parasitoids, cleptoparasites or even facultative or obligatory seed predators (Pereira et al. 2007; Wang et al. 2014).

The Sycophaginae are NPFW that occur in all tropical regions. They are associated with Ficus subgenera Urostigma and Sycomorus (Cruaud et al. 2011a; Wiebes 1966). Six genera and ca. 74 described species belong to the Sycophaginae (Cruaud et al. 2011b; Farache et al. 2013; Farache \& Rasplus 2014; Farache \& Rasplus 2015). However, the overall diversity of the Sycophaginae is estimated to ca. 700 species (Cruaud et al. 2011b).

Bouček (1988) assigned all NPFW subfamilies (namely Epichrysomallinae, Otitesellinae, Sycoecinae, Sycophaginae and Sycoryctinae), and pollinators to Agaonidae, mostly based on the morphology of the postgenal bridge. However, molecular phylogenetic analyses and a reevaluation of the postgenal bridge morphology evidenced this grouping as non-monophyletic (Rasplus et al. 1998). Heraty et al. (2013) recovered Sycophaginae as sister to the pollinating fig 
62 wasps (Agaonidae) and proposed the inclusion of Sycophaginae in Agaonidae; most of the other 63 fig wasp subfamilies were assigned to Pteromalidae.

64 Life history traits and oviposition behaviour are variable in Sycophaginae. Most species 65 oviposit through the fig wall and induce galls in pistilate flowers. They also may oviposit in galls 66 induced by other wasps and develop as cleptoparasites or parasitoids. Several species (a clade 67 within Sycophaga) enter the fig through the ostiole as pollinators do, and induce galls in pistilate 68 flowers (Cook \& Rasplus 2003; Cook \& Segar 2010; Cruaud et al. 2011b; Elias et al. 2012; Galil 69 et al. 1970).

Two genera of Sycophaginae are associated with Ficus in the Neotropical region, namely Anidarnes Bouček, 1993 and Idarnes Walker, 1843 (Bouček 1993; Rasplus \& Soldati 2005). 72 They are strictly associated with Ficus section Americanae. Idarnes is the most diverse NPFW 73 genus in the Neotropics. Twenty-three species are recognised as belonging to Idarnes (Bouček 1993; Cruaud et al. 2011b; Gordh 1975), but the overall diversity of the genus is estimated to nearly 300 species (Cruaud et al. 2011b). Some Old-World species were classified under Idarnes, however they all belong to Sycophaga Westwood, 1840 (=Apocryptophagus Ashmead, 1904) (Bouček 1993; Cruaud et al. 2011b; Gordh 1975) and consequently, Idarnes is restricted to the Neotropics. Concerning nomenclature, Idarnes should be treated as masculine as well as Anidarnes and other derived names (Farache et al. 2013). The name probably refers to an eminent Persian commander, Hydarnes, who was given command of the "Immortals" and fought the Greeks in the battle of Thermopylae, $480 \mathrm{BC}$.

Three morphological species-groups of Idarnes are recognised, namely I. carme, I. flavicollis and I. incertus species-groups. They exhibit clear morphological differences (Bouček 1993) and contrasted life history traits. Species belonging to the I. incertus species-group are gall-makers and oviposit before pollination. Idarnes flavicollis species-group species are also gall-makers but oviposit at the same time as pollinators. The species belonging to the I. carme species-group oviposit after pollination and are probably cleptoparasites associated with pollinator's larvae (Elias et al. 2008; Elias et al. 2012).

The purpose of this paper is to provide a taxonomic revision of the Idarnes incertus species-group. Three species are re-described and 17 species are described from samples collected in Brazil, Costa Rica and Colombia. All species are illustrated and an identification key 
93 and eight outgroups were inferred using multiple genes, and their relationships were discussed in

94 the light of the taxonomy of their hosts.

95

96 Materials \& Methods

97

98

Specimen collection and morphological study.

99 Figs were sampled before maturity and transferred to tissue bags until wasp emergence.

100 Wasps were killed using ethyl acetate or freezing and stored in $70 \%$ ethanol. Geographical 101 coordinates and altitude were recorded in the field using a GPS device or estimated using label 102 information. ICMBio provided permissions for material sampling to RASP in Brazil (Permit $103 \# 1870297)$.

104 Specimens were dehydrated through an ethanol and HMDS series (Heraty \& Hawks 1998) 105 or critical point dried (Gordh \& Hall 1979) using BALTEC CPD 030'. Insects were card106 mounted following Noyes (1982). Morphological terminology follows Gibson (1997). 107 Measurements were taken using Leica application suite V3.6. Abbreviations for measurements 108 used in the text include: POL $=$ distance between lateral ocelli; OOL $=$ distance between one 109 posterior ocellus and adjacent composite eye.

110 Multi-entry online keys were produced using Lucid ${ }^{\circledR}$ v. 3.3. They are available at figweb 111 (www.figweb.org - van Noort \& Rasplus 2016) and as supplementary material 1.

112 The electronic version of this article in Portable Document Format (PDF) will represent a 113 published work according to the International Commission on Zoological Nomenclature (ICZN), 114 and hence the new names contained in the electronic version are effectively published under that 115 Code from the electronic edition alone. This published work and the nomenclatural acts it 116 contains have been registered in ZooBank, the online registration system for the ICZN. The 117 ZooBank LSIDs (Life Science Identifiers) can be resolved and the associated information viewed 118 through any standard web browser by appending the LSID to the prefix http://zoobank.org/. The 119 LSID for this publication is: urn:lsid:zoobank.org:pub:22286699-8306-4931-8D7F$1207 B F 05 E B 2 B 304$. The online version of this work is archived and available from the following 121 digital repositories: PeerJ, PubMed Central and CLOCKSS.

122

123 Acronyms for repositories follow Arnett et al. (1993) when available: 
124

125

126

127

128

129

130

131

132

133

134

135

136

137

138

139

140

141

142

143

144

145

146

147

148

149

150

151

152

153 154 protein translations to detect frameshift mutations and premature stop codons using MEGA 4

BMNH: The Natural History Museum, London, U.K.

CBGP: Centre de Biologie pour la Gestion des Populations, Montferrier-sur-Lez, France.

EBCR: Escuela de Biologia, Universidad de Costa Rica, San José, Costa Rica

MZSP: Museu de Zoologia da Universidade de São Paulo, São Paulo, Brazil.

NMW: Naturhistorisches Museum, Wien, Austria.

SAMC: Iziko South African Museum, Cape Town, South Africa.

RPSP: Universidade de São Paulo, Ribeirão Preto, Brasil.

USNM: National Museum of Natural History, Washington D.C., U.S.A.

2

3 Illustrations. Images of specimens were produced with a Leica MZ16 stereoscope connected to a digital camera and a computer workstation running Leica Application Suite (LAS) V3.6 imaging software. Image series comprising about 15-20 focal planes were merged to produce a single image with increased depth of field.

Some specimens were dissected, mounted and sputter-coated with gold for scanning electron microscopy (SEM), which was performed with a Zeiss EVO 50 microscope. SEM images of species with few specimens were obtained with a low vacuum protocol.

\section{Molecular protocols and phylogenetic analyses}

In this study, we amplified one nuclear protein-coding region (F2 copy of elongation factor-1 $\alpha, E F-1 \alpha$ ), two mitochondrial protein-coding regions (Cytochrome Oxydase I - COI and Cytochrome B $-C y t B$ ), and two regions of the $r R N A 28 S$ (D2-D3 and D4-D5 expansion regions). DNA extraction, PCR conditions, and sequencing protocols follow Cruaud et al. (2010) and Cruaud et al. (2011a). Forward and reverse strands for each fragment were assembled using the software Geneious v.6.1.8. All the sequences were deposited in GenBank (accession numbers in supplementary material 2). Our dataset consisted of 33 terminals, comprehending 25 specimens for 13 ingroup species belonging to the Idarnes incertus species-group and eight outgroup species, representing all other Idarnes species-groups, all known Sycophaginae genera, and an Epichrysomallinae genus (Pteromalidae).

52 Sequence alignment for all markers was performed in MAFFT v. 7 (Katoh \& Standley 53 2013) using the L-INS-i algorithm, and visually inspected. In protein coding genes, we checked 
155 (Kumar et al. 2008). The most appropriate model of sequence evolution for each data subset 156 most likely to have experienced similar evolutionary processes (mitochondrial genes, EF-1 $\alpha$, 157 rRNA 28S) was identified using Akaike information criterion (Akaike 1973) as implemented in 158 jModeltest v. 2.1.7 (Darriba et al. 2012; Guindon \& Gascuel 2003). Since we used multiple loci 159 to infer phylogenetic relationships, we established different partitions for each locus included in 160 the analyses, allowing parameters to vary among partitions.

161 Phylogenetic analyses were performed using maximum likelihood (ML) and Bayesian 162 methods, conducted in the CIPRES Science Gateway (Miller et al. 2010).

163 Partitioned ML analyses were performed using RAxML v 8 (Stamatakis 2014), and the 164 GTRCAT approximation was used for performing associated bootstrapping (1000 replicates). 165 Bootstrap percentage $\left(\mathrm{ML}_{\mathrm{BP}}\right)>95 \%$ was considered as strong support and a $\mathrm{ML}_{\mathrm{BP}}<70 \%$ as 166 weak.

167 Bayesian phylogenetic analyses were conducted using MrBayes v. 3.2.2 (Ronquist et al. 168 2012). We assumed across-partition heterogeneity in model parameters by considering the 169 parameter $\mathrm{m}$. Parameter values were initiated with default priors; branch lengths were estimated 170 using default exponential priors. The optimization of the posterior probability was achieved 171 using Metropolis-coupled Markov chain Monte Carlo (MCMC). To improve mixing of the cold 172 chain and avoid converging on local optima, we executed two independent runs including a cold 173 chain and three incrementally heated chains for each run. The heating parameter was set to 0.02 174 in order to allow more frequent swapping between cold and heated chains. The runs were 175 executed for 10 million generations, and values were sampled every 1000 generations. A 176 NEXUS file including gene alignment and MrBayes block is included as supplementary material 177 3. We also ensure the convergence between parameters from the two chains by analysing 178 estimates and frequency distributions of each parameter using Tracer v. 1.5 (Rambaut et al. 179 2013). We examined the plot of overall model likelihood against generation number to find the 180 point where the likelihood started to fluctuate around a constant value, and applied a $10 \%$ 181 relative burn-in. The results were based on the pooled samples from the stationary phases of the 182 two independent runs. Posterior probabilities (PP) $>0.95$ were considered as strong support. 183

\section{Results}


186

187

188

189

190

191

192

193

194

195

196

197

198

199

200

201

202

203

204

205

206

207

208

209

210

211

212

213

214

2154 Postmarginal vein shorter than stigmal vein. Head sculpture homogeneous. Antenna with 2 216

\section{Morphological definition; key to genera and species-groups}

Idarnes is the sister group of Sycophaga, a diversified Old World genus mostly associated with Ficus subgenenus Sycomorus, but two species are associated with $F$. subg. Urostigma. Idarnes carme species-group is sister to a clade grouping I. flavicollis species-group and $I$. incertus species-group (Cruaud et al. 2011a; Cruaud et al. 2011b).

Sycophaga and all Idarnes species-groups can be identified using the following key:

1 Body smooth, sculpturation shallow. Notaulus, axillula, frenal sulcus and other sutures without obvious crenulation. Head flattened dorsoventrally (Fig. 1A). Oviposits internally in figs

Old World, Sycophaga Westwood, 1840 (part)

--- Body sculpture at least slightly reticulate. Notaulus, axillula, frenal sulcus and other sutures at least slightly crenulated. Head globose or subglobose in lateral view (Fig. 1B). Oviposits through the fig wall

2

2 Malar sulcus present (Fig. 1B). Antenna with two anelli and postmarginal vein longer than stigmal vein.

...Old World, Sycophaga (part, formerly Apocryptophagus)

--- Malar sulcus absent. Antenna with one anellus or, if two anelli (I. flavicollis and I. incertus species-groups) postmarginal vein shorter than stigmal vein (only exception known is the fossil species Idarnes thanatos Farache \& Rasplus, 2016).

.New World, Idarnes. 3

3 Body mostly without metallic tinge (Figs 2, 3). Ovipositor sheaths shorter than body length. Funicular segments transverse. I. incertus species-group

--- Body with metallic tinge, ovipositor as long as body or longer. Funicular segments nearly as long than wide or longer than wide 4 anelli (Fig. 1C). Mandibles tridentate. Clypeal margin bilobed (Fig. 1D) (trilobed in $I$. 
--- Postmarginal vein longer than stigmal. Head sculpture stronger near vertex Antenna with one anellus (Fig. 1E). Mandibles bidentate. Clypeal margin usually straight or unilobed (excepted one undescribed species) (Fig. 1F)

I.

223

224 carme species-group

The I. incertus species-group shares similarities with Anidarnes Bouček, 1993 - another neotropical Sycophaginae genus - but can be distinguished by the following characters: (1)

226 antennae usually inserted closer to the clypeal margin than to the median ocellus, or at most at

227 the same distance, whereas in Anidarnes they are inserted closer to the median ocellus; (2) 228 ovipositor without the median constriction apomorphic of Anidarnes (to the exception of $A$. 229 dissidens Farache \& Rasplus 2013); (3) metascutellum at least 3× as wide as long in incertus 230 species-group whereas at most $2 \times$ as wide as long, or trapezoidal, in Anidarnes (sometimes the 231 metascutellum is inconspicuous in both groups). A key to the genera of neotropical fig wasps is 232 provided by Bouček (1993) and Rasplus \& Soldati (2005).

Idarnes incertus species-group

235

236

1993 Bouček, Z., Journal of Natural History 27: 200-203 - species-group treatment for Idarnes.

238

\section{Description}

240 Females. Body length 1.3-2.8 mm. Ovipositor length 0.4-1.6mm. Body colour yellow to black, 241 metallic tinge mostly absent. Wings hyaline. Head transverse (1.2-1.4× as wide as high). Face 242 sculpture reticulate. Malar sulcus absent. Maxillary and labial palpi composed at most of two or 243 three segments, the last one frequently reduced and setae-like. Clypeal margin bilobed. Frontal 244 depression (scrobal cavity) shallow, rarely including median ocellus. Supraclypeal area delimited 245 laterally by subantennal grooves. Antennae inserted closer to clypeal margin than to median 246 ocellus (sometimes nearly equidistant from them). Toruli separated by one torulus diameter or 247 less, but never closer than $0.5 \times$ torulus diameter. Antenna with 12-13 antennomeres (11-12 
248 flagellomeres; one or two anelli) and a very small terminal protuberance. Clava not well 249 delimited. Vertex slightly concave. POL 2.2-3.4× OOL. Mesosoma slightly curved dorsally. 250 Pronotum $0.7-1.0 \times$ as long as mesoscutum. Notaulus complete and usually crenulated. 251 Mesoscutellum 1.1-1.5× as long as wide near transscutal articulation. Metascutellum transverse, 252 at most as long as frenum, sometimes inconspicuous. Postmarginal vein very short, at most $0.5 \times$ 253 as long as stigmal vein. Ovipositor sheaths shorter than body length, and $0.8-3 \times$ as long as hind 254 tibia.

255

256 Males. Similar to females, sometimes showing variation in tinge/colour and body proportions 257 (see supplementary material 4). Flagellum shorter than in females. Wings medially infuscated, 258 with dense pilosity, especially at the costal and apical margin, and near marginal vein.

259

260 Remarks. Idarnes is treated here as masculine as explained in Farache et al. (2013).

Key to species

263

264

265

The key is based on female characters. I. brasiliensis (Mayr 1906) was not included since only one male could be analysed.

266

2671 Body completely black or dark brown (Figs 2G, 3C, E), including pronotum (Figs 8G, 9C, 268

269 E) and propodeum (Figs 10G, 11C, E). Scrobal depression and face engraved to

270 reticulated (Figs 4G, 5C, E). propodeum yellow (as Figs 10C, H, $11 \mathrm{~J}$ ). Scrobal depression mostly smooth, face

273

2742 Mesoscutum and scutellar-axillar complex strongly curved in lateral view (Fig. 3C). 275 Supraclypeal area narrower than torulus (Fig. 5C). Frenal sulcus crenulated and

276

277 conspicuous (Fig. 13C). Metascutellum nearly as long as, or longer than frenum (Fig. 13C). Ex F. americana subsp. andicola. 
279 --- Mesoscutum and scutellar-axillar complex not strongly curved in lateral view (Figs 2G, $2803 \mathrm{E}$ ). Supraclypeal area as wide as, or wider than torulus (Figs 4G, 5E). Frenal sulcus not 281 crenulated (Figs 12G, 13E). Metascutellum inconspicuous in dorsal view (Figs 12G,

283

2843 Frons becoming yellowish near clypeus. Supraclypeal area as wide as torulus (Fig. 4G). 285 Antenna with two anelli (Fig. 6G). Postmarginal vein nearly absent (Fig. 14G). Ex F. hartwegii.

I. comptoni sp. $\mathrm{n}$.

287 --- Frons completely black. Supraclypeal area wider than torulus (Fig. 5E). Antenna with one

288

289 anellus (Fig. 7E). Postmarginal vein nearly as long as $0.5 \times$ stigmal vein length (Fig. 15E). Ex F. hartwegii.

\section{I. hansoni Bouček, 1993}

290

2914 Head and mesosoma brown black in lateral view; pronotum yellow (Figs 2B, F, H, 3A), at least laterally, or propodeum yellow dorsally (Figs 10C, D, H). .5

293 --- Head and mesosoma predominantly yellow in lateral view (as in Figs 2A, D, E)..

294 9

295

296

5 Axillula reticulated, without longitudinal striae (Fig. 13A). Notauli not crenulated (Fig.

297 13A). Ex F. americana.

\section{I. flavicrus sp. $\mathbf{n}$.}

298 --- Axillula longitudinally striated (Figs 12B, C, F, H). Notauli at least slightly crenulated

299 (Figs 12B, C, F, H).

\section{.6}

300

301

6 Propodeum dorsally yellow (Figs 10C, H). Frenal sulcus conspicuous (Figs 12C, H). Body

302 length $1.8-2.1 \mathrm{~mm}$. 7

303 --- Propodeum dorsally brown (Figs 10B, F). Frenal sulcus inconspicuous (Figs 12B, F). Body 304 length $1.3-1.6 \mathrm{~mm}$. .8

305

306

7 Pronotum laterally brown. Legs brown. Ovipositor 1.1-1.3× hind tibia length (Fig. 2C). Ex

308 --- Pronotum laterally yellow. Legs predominantly yellow. Ovipositor $1.5 \times$ hind tibia length 
3118 Hind coxae yellow (Fig. 2B). Propodeal median line absent (Fig. 12B). Ex F. americana subsp. guianensis form mathewsii. I. amazonicus sp. $\mathbf{n}$.

313 --- Hind coxae brown (Fig. 2F). Propodeal median line present as a faint longitudinal reticulation (Fig. 12F). Ex F. colubrinae.

I. brunneus sp. $\mathrm{n}$.

315

3169 Ovipositor nearly $2.5 \times$ as long as hind tibia or longer (Figs $3 \mathrm{~B}, \mathrm{D}, \mathrm{G}) \ldots \ldots \ldots \ldots . .10$

317 --- Ovipositor as long as or shorter than $2 \times$ hind tibia (as in Figs 2I, 3I, J)............12

318

319

10 Metasoma laterally yellow (Fig. 3B), dorsally with brown black transversal stripes. Frenal

320 sulcus conspicuous (Fig. 13B). Ex F. aurea form isophlebia.

321

I. flaviventris sp. $\mathrm{n}$.

322 --- Metasoma black (Figs 3D, G). Frenal sulcus inconspicuous, sparsely crenulated (Figs 13D,

323

G). 11

324

32511 Scutellar-axillar complex dorsally black (Fig. 11G). Supraclypeal area narrower than torulus (Fig. 3G). Anterior margin of propodeum angulose medially (Fig. 11G). First funicular segment with 12-17 multiporous late sensilla (Fig. 7G). Ex F. obtusifolia.

\section{I. maximus sp. $\mathrm{n}$.}

12 First funicular segment with more than four multiporous plate sensilla (as in Figs 6E, I, 7I,

--- First funicular segment with four our less multiporous plate sensilla (Figs 6A, D). 337

33813 Ovipositor as long as, or shorter than hind tibia (Figs 2E, I).

339 --- Ovipositor longer than hind tibia (as in figs 3H, I). 
34114 Metasoma laterally yellow (Fig. 2E). Subantennal groove as long as torulus (Fig. 4E).

342 Supraclypeal area wider than torulus (Fig. 4E). Ex F. citrifolia.

344 --- Metasoma entirely black (Fig. 2I). Subantennal groove longer than torulus (Fig. 4I).

345 Supraclypeal area as wide as torulus (Fig. 4I). Ex F. citrifolia.

346

.I. dimorphicus sp. $\mathrm{n}$.

347

34815 Ovipositor $1.8-2 \times$ as long as hind tibia (Figs $3 \mathrm{H}$, I). Supraclypeal area narrower than 349 torulus (Figs $5 \mathrm{H}, \mathrm{I})$. 16

350

Ovipositor $1 \times$ to $1.5 \times$ as long as hind tibia (Figs $3 \mathrm{~F}, \mathrm{~J}$ ). Supraclypeal area as wide as torulus or wider (Fig. 5J). 17

352

35316 Metasoma ventrally yellow (Fig. 3I). Distance from torulus to median ocellus $1.4 \times$ distance from torulus to oral margin (Fig. 5I). Antenna with one anellus. Ex F. crocata.

356 --- Metasoma ventrally brown-black (Fig. 3H). Distance from torulus to median ocellus $0.9 \times$ distance from torulus to oral margin (Fig. 3H). Antenna with 2 anelli. F. aurea form isophlebia.

\section{I. nigriventris sp. $\mathrm{n}$.}

17 Scutellar-axillar complex smoky yellow, propodeum more yellow (Fig. 11J). Metasoma brown black (Fig. 3J). POL $3 \times$ OOL Ex F. americana subsp. americana.

363 --- Scutellar-axillar complex yellow, nearly same colour as propodeum (Fig. 11F). Metasoma brown black, first tergite and ventral region yellow (Fig. 3F). POL 2.2× OOL Ex $F$. aurea \& F. citrifolia.

18 Head and mesosoma dorsally brown, slightly metallic green (Fig. 8D). Frenal sulcus

--- Head and mesosoma yellow (Fig. 8A). Frenal sulcus conspicuous (Fig. 12A). Ex $F$. 


\section{Species descriptions}

374

375

376

Idarnes amacayacuensis Farache \& Rasplus, sp. n.

377

urn:Isid:zoobank.org:act:48D01597-E7B0-41AC-8A7E-DCE21AA97EE6

378

379

(Figs 2A, 4A, 6A, 8A, 10A, 12A, 14A)

Type material. Holotype: $q$, COLOMBIA: Leticia: PN Amacayacu, $-3.30^{\circ},-70.14^{\circ}, 130 \mathrm{~m}$, 381 20.XI.1993, Lachaise D., ex Ficus pertusa (CBGP).

Paratypes: COLOMBIA: Leticia: PN Amacayacu, $-3.30^{\circ},-70.14^{\circ}, 130 \mathrm{~m}, 6 \stackrel{+}{\circ}, 5 \overbrace{}^{\Uparrow}, 20 . X \mathrm{XI} .1993$, Lachaise D., ex Ficus pertusa (4ๆ $4 \hat{\jmath}$ CBGP, $2 \propto 1 \hat{\jmath}$ RPSP).

384

Etymology. The specific name refers to the type locality, the Amacayacu National Natural Park 386 in Colombia.

Diagnosis ( + ). Head, antennae, mesosoma, and legs yellow. Metasoma dark brown. 389 Metascutellum inconspicuous in dorsal view. Propodeal median line present, traceable at least in 390 the anterior half of propodeum. Ovipositor sheaths $1.6 \times$ as long as hind tibia.

\section{Female.}

393 Size and colour. Body length $1.5 \mathrm{~mm}$. Ovipositor length $0.5 \mathrm{~mm}$. Head, antennae, mesosoma, 394 and legs yellow. Metasoma dark brown.

395 Head. Supraclypeal area narrower than torulus. Subantennal groove as long as torulus. Distance 396 from torulus to median ocellus $1.3 \times$ distance from torulus to oral margin. POL $3.0 \times$ OOL. Scape $3971.9 \times$ as long as pedicel. Antenna with two anelli (character sometimes inconspicuous). First 398 funicular segment $0.7 \times$ as long as wide, with 1-3 multiporous plate sensilla.

399 Mesosoma. Mesoscutum reticulate. Mesoscutum and scutellar-axillar complex not strongly 400 curved in lateral view. Notaulus crenulated. Mesoscutellum $1.2 \times$ as long as wide near transcutal 401 articulation. Axillula with longitudinal striae. Frenal sulcus barely crenulated, conspicuous. 402 Metascutellum inconspicuous in dorsal view. Anterior margin of propodeum angulose medially. 
403 Propodeal median line present, traceable at least in the anterior half of propodeum. Stigmal vein $4040.9-1 \times$ as long as marginal vein. Stigmal vein without adstigmal setae. Postmarginal vein very 405 short, as long as $1 / 3 \times$ stigmal vein length.

406 Metasoma. Ovipositor sheaths $1.6 \times$ as long as hind tibia.

407

408 Male. Similar to female. Body colour paler. Mesoscutellum infuscated. Distance from torulus to 409 median ocellus $1.5 \times$ distance from torulus to oral margin. POL $2.3 \times$ OOL. First funicular 410 segment $0.5 \times$ as long as wide.

411

412 Host plant. Ficus pertusa Linnaeus filius.

413

414 Idarnes amazonicus Farache \& Rasplus, sp. n.

415 urn:1sid:zoobank.org:act:6F44A1B2-73CC-4267-9F02-AF4E7FF600BC

416 (Figs 2B, 4B, 6B, 8B, 10B, 12B, 14B)

417

418 Type material. Holotype: + , BRAZIL: Amazonas: São Gabriel da Cachoeira, Igarapé da 419 Cachoeirinha, -3.09853 ${ }^{\circ}-59.97624^{\circ}$, 19.XI.2007, Santos O.A., ex Ficus americana subsp. 420 guianensis form mathewsii $\mathrm{n}^{\mathrm{o}} \mathrm{JRAS} 02147$ 03 (MZSP).

421 Paratypes. BRAZIL: Amazonas: São Gabriel da Cachoeira, Igarapé da Cachoeirinha, -3.09853º $422-59.97624^{\circ}, 2$, $19 . X I .2007$, Santos O.A., ex Ficus americana subsp. guianensis form mathewsii 423 no JRAS02147_03 (1q CBGP, 1q RPSP).

424

425

Etymology. The specific name refers to the province where the type was collected.

426

427 Diagnosis (†). Body predominantly brown black. Pronotum and propodeum slightly yellow. 428 Legs yellow, femora slightly brown. Supraclypeal area as wide as torulus, or slightly wider. 429 Subantennal groove slightly longer than torulus. Distance from torulus to median ocellus $1 \times$ 430 distance from torulus to oral margin. Metascutellum inconspicuous to about $0.5 \times$ as long as 431 frenum in dorsal view. Ovipositor sheaths $1.3 \times$ as long as hind tibia.

432

433 Female. 
434 Size and colour. Body length 1.4-1.5 mm. Ovipositor length $0.4 \mathrm{~mm}$. Predominantly brown 435 black. Lower face yellow. Scape and pedicel yellow, pedicel slighly brown. Pronotum laterally 436 yellow. Propodeum slightly yellow near its posterior margin. Legs yellow, femora slightly brown. 437 Head. Supraclypeal area as wide as torulus. Subantennal groove as long as torulus. Distance 438 from torulus to median ocellus $1 \times$ distance from torulus to oral margin. POL $2.5 \times$ OOL. Scape $4392.3 \times$ as long as pedicel. Antenna with two anelli (character sometimes inconspicuous). First 440 funicular segment $0.5 \times$ as long as wide, with 6-9 multiporous plate sensilla.

441 Mesosoma. Mesoscutum reticulate. Mesoscutum and scutellar-axillar complex not strongly 442 curved in lateral view. Notaulus with shallow crenulation. Mesoscutellum $1.4 \times$ as long as wide 443 near transscutal articulation. Axillula longitudinally striate to reticulate. Frenal sulcus barely 444 crenulated and inconspicuous. Metascutellum inconspicuous to approximately $0.5 \times$ as long as 445 frenum in dorsal view. Anterior margin of propodeum concave medially. Propodeal median line 446 inconspicuous. Stigmal vein $0.7 \times$ as long as marginal vein, with 3 adstigmal setae. Postmarginal 447 vein very short, as long as $1 / 3 \times$ stigmal vein length.

448 Metasoma. Ovipositor sheaths $1.3 \times$ as long as hind tibia.

449

450 Male. Unknown.

451

452 Host plant. Ficus americana Aublet subsp. guianensis (Desvaux) Berg form mathewsii (Miquel) 453 Berg.

454

455

456

Idarnes americanae Farache \& Rasplus, sp. n.

457 (Figs 2C, 4C, 6C, 8C, 10C, 12C, 14C)

458

459 Type material. Holotype: + , COSTA RICA: La Fortuna: Arenal, $10.49916^{\circ}-84.71019^{\circ}$, 460 18.IV.2010, Cruaud A. \& Rasplus J.Y., ex Ficus americana no JRAS02841_01 (CBGP).

461

462 Paratypes. COSTA RICA: Heredia: Santo Domingo, 8.43 ${ }^{\circ}-83.41^{\circ}, 4 \uparrow, 3 \hat{\jmath}, 15 . X I .2002$, 463 Hanson P., ex. Ficus costaricana $n^{\circ}$ JRAS01364 (3q $2 \hat{\jmath}$ CBGP, $1 q 1 \lesssim$ RPSP); La Fortuna: 
464 Arenal, 10.49916 ${ }^{\circ}-84.71019^{\circ}, 4, 3 \hat{\jmath}, 18 . I V .2010$, Cruaud A. \& Rasplus J.Y., ex Ficus

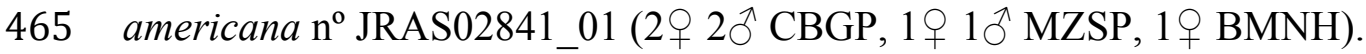

466

467 Etymology. The specific name refers to the Ficus section to which Idarnes is associated with.

468

469 Diagnosis ( $९$ ). Body colour and legs predominantly brown. Pronotum and propodeum mostly 470 yellow. Supraclypeal area narrower than torulus. Subantennal groove shorter than torulus. 471 Distance from torulus to median ocellus $1.2 \times$ distance from torulus to oral margin. Mesoscutum 472 medially with longitudinal striae. Metascutellum nearly $0.5 \times$ as long as frenum or shorter in 473 dorsal view. Ovipositor sheaths $1.1-1.3 \times$ as long as hind tibia.

474

475 Female.

476 Size and colour. Body length 1.9-2.1 mm. Ovipositor length 0.5-0.6 mm. Predominantly brown. 477 Scape yellow. Pedicel yellow brown, flagellum brown. Pronotum laterally yellow. Lateral panel 478 of metanotum brown black. Propodeum yellow. Legs brown, fore coxa and tarsi yellow. Fore 479 tibia yellow brown.

480 Head. Supraclypeal area narrower than torulus. Subantennal groove shorter than torulus. 481 Distance from torulus to median ocellus $1.2 \times$ distance from torulus to oral margin. POL $3.4 \times$ 482 OOL. Scape $2.3 \times$ as long as pedicel. Antenna with two anelli (character sometimes 483 inconspicuous). First funicular segment $0.7 \times$ as long as wide, with $7-9$ multiporous plate sensilla. 484 Mesosoma. Mesoscutum reticulate to punctate reticulate, medially with longitudinal striae. 485 Mesoscutum and scutellar-axillar complex not strongly curved in lateral view. Notaulus 486 crenulated. Mesoscutellum $1.3 \times$ as long as wide near transscutal articulation. Axillula with 487 longitudinal striae. Frenal sulcus crenulated and conspicuous. Metascutellum nearly $0.5 \times$ as long 488 as frenum or shorter in dorsal view. Anterior margin of propodeum angulose medially. Propodeal 489 median line present, traceable at least in the anterior half of propodeum. Stigmal vein as long as 490 marginal vein, with $2-3$ adstigmal setae. Postmarginal vein very short, as long as $1 / 3 \times$ stigmal 491 vein length.

492 Metasoma. Ovipositor sheaths $1.1-1.3 \times$ as long as hind tibia. 493

494 Male. Body predominantly yellow. Lateral panel of metanotum yellow brown. Metasoma brown, 
495 yellow at the margin of tergites. Distance from torulus to median ocellus $1.5 \times$ distance from 496 torulus to oral margin. POL $3.1 \times$ OOL. First funicular segment $0.5 \times$ as long as wide.

497

498 Host plant. Ficus americana subsp. americana Aublet and Ficus costaricana (Liebmann) 499 Miquel.

500

501

Idarnes badiovertex Farache \& Rasplus, sp. n.

502 urn:lsid:zoobank.org:act:28673472-103B-4576-A41E-4851E4194771

503 (Figs 2D, 4D, 6D, 8D, 10D, 12D, 14D)

504

505 Type material. Holotype: + , COLOMBIA: Leticia: PN Amacayacu, $-3.30^{\circ}-70.14^{\circ}, 130 \mathrm{~m}$, 506 20.XI.1993, Lachaise D., ex Ficus pertusa (CBGP).

507

508 Paratypes: COLOMBIA: Leticia: PN Amacayacu, $-3.30^{\circ}-70.14^{\circ}, 130 \mathrm{~m}, 16 \bigcirc, 1 \overbrace{}^{\Uparrow}, 20 . X I .1993$, 509 Lachaise D., ex Ficus pertusa (13ํ 1ð CBGP, 3 ㅇ RPSP).

510

511

512

Etymology. The specific name refers to the brown colouration of the top of the head.

513 Diagnosis ( $(+)$. Head yellow, dorsally brown. Mesosoma dorsally brown black, axillula slightly 514 metallic green. Propodeum yellow. First funicular segment with 1-2 multiporous plate sensilla. 515 Ovipositor sheaths ca. $1.8 \times$ as long as hind tibia.

516

517 Female.

518 Size and colour. Body length $1.3 \mathrm{~mm}$. Ovipositor length $0.5 \mathrm{~mm}$. Head and mesosoma yellow.

519 Antennae yellow. Head dorsally brown, slightly metallic green. Mesosoma dorsally brown black, 520 axillula slightly metallic green. Propodeum dorsally yellow. Legs yellow. Metasoma brown.

521 Head. Supraclypeal area as wide as torulus. Subantennal groove shorter than torulus. Distance 522 from torulus to median ocellus $1.4 \times$ distance from torulus to oral margin. POL $3 \times$ OOL. Scape $5231.7-1.8 \times$ as long as pedicel. Antenna with two anelli (character sometimes inconspicuous). First 524 funicular segment $0.5 \times$ as long as wide, with 0-2 multiporous plate sensilla.

525 Mesosoma. Mesoscutum reticulate. Mesoscutum and scutellar-axillar complex not strongly 
526 curved in lateral view. Notaulus with shallow crenulation. Mesoscutellum $1.4 \times$ as long as wide 527 near transcutal articulation. Axillula with longitudinal striae. Frenal sulcus smooth and faint.

528 Metascutellum inconspicuous in dorsal view. Anterior margin of propodeum angulose medially.

529 Propodeal median line inconspicuous. Stigmal vein $0.9 \times$ as long as marginal vein, with $3-4$

530 adstigmal setae. Postmarginal vein nearly absent, shorter than $1 / 5 \times$ stigmal vein length.

531 Metasoma. Ovipositor sheaths ca. $1.8 \times$ as long as hind tibia.

532

533 Male. Similar to female. Body colour paler.

534

535 Host plant. Ficus pertusa Linnaeus filius.

536

537 Idarnes brasiliensis (Mayr, 1906) (comb. nov.)

538 (Figs in supplementary material 5)

539

5401906 Mayr, G. Entomologische Zeitung Wien 25:185. Description (우) (Comb. Sycophila 541 brasiliensis).

542

543 Type material. Lectotype (here designated) BRAZIL: Santa Catarina: Blumenau, 1ڤ̂, [no 544 date], Fritz Müller, ex Ficus doliaria (=F. gomelleira) (NMW).

545

546 Diagnosis $(\overbrace{}^{\Uparrow})$. Body colour predominantly yellow orange. Mesosoma $1.4 \times$ as long as wide.

547 Axillula longitudinally striated. Frenal sulcus inconspicuous. Metascutellum inconspicuous in 548 dorsal view. Propodeal median line present, conspicuous. Postmarginal vein very short, as long 549 as $1 / 3 \times$ stigmal vein length.

550

551 Female: Described by Mayr (1906), but we could not find any female specimens at NMW.

552

553 Host plant. Ficus gomelleira Kunth \& Bouché.

554

555 Remarks: There is only one male specimen collected by Mayr at NMW, minuten-mounted and 556 decapitated. Despite the absence of head, the following characters ascertain its position within 
557 the Idarnes incertus species-group: (1) Winged male, (2) body colour, (3) postmarginal vein 558 compared to stigmal vein, (4) shape of mesoscutellum and (4) striated axillula.

559

560 Idarnes brevis Farache \& Rasplus, sp. n.

561 urn:1sid:zoobank.org:act:06317A88-E1C5-48AB-83FB-66E79424360C

562 (Figs 2E, 4E, 6E, 8E, 10E, 12E, 14E)

563

564 Type material. Holotype: + , COSTA RICA: San José: Santiago de Puriscal, 9.84132 $56584.31540^{\circ}, 2 . I .2007$, Fernandez, ex Ficus citrifolia n ${ }^{\circ}$ JRAS01954_02 (CBGP).

566

567 Paratypes. COSTA RICA: Alajuela: San Ramon, Piedades Sur $10.11^{\circ}-84.53^{\circ}, 23 \bigcirc, 16 \diamond^{\lambda}$, 568 5.I.2008, Vasquez J., ex Ficus citrifolia n ${ }^{\circ}$ JRAS03857 (20 15 ๙ CBGP, 391 RPSP); 569 Heredia: Santo Domingo, 9.988886 $-84.083926^{\circ}, 5 \circ$, X.2005, Hanson P., ex Ficus hemsleyana 570 (=F. citrifolia $) n^{\circ}$ JRAS01530_02 (1q CBGP, $2 q$ RPSP, $1 q$ MZSP, $1 q$ SAMC); San José: 571 Santiago de Puriscal, 9.84132 $-84.31540^{\circ}, 1 q, 7{ }^{\circ}, 2 . I .2007$, Fernandez, ex Ficus citrifolia $\mathrm{n}^{\circ}$ 572 JRAS01954_02 (1q7ð CBGP), Univ. San José, Est. Fabio B. Moreno, 9.944526 -84.091639, 573 3q, 5.III.2008, Rasplus J.Y. \& Ramírez W., ex Ficus hemsleyana $(=$ F. citrifolia $) \mathrm{n}^{\mathrm{o}}$ 574 JRAS02284_03 (CBGP).

575

576 Etymology. The specific name refers to the short ovipositor.

577

578 Diagnosis ( + ). Predominantly yellow orange. Metasoma dorsally brown black, mostly at the 579 margin of tergites. Supraclypeal area wider than torulus. Subantennal groove as long as torulus. 580 Distance from torulus to median ocellus $0.9 \times$ distance from torulus to oral margin. First funicular 581 with 12-15 multiporous plate sensilla. Ovipositor sheaths $0.8-0.9 \times$ as long as hind tibia.

582

\section{Female.}

584 Size and colour. Body length 2.1-2.3 mm. Ovipositor length 0.4-0.5 mm. Predominantly yellow 585 orange. Metasoma dorsally brown black, mostly at the margin of tergites.

586 Head. Supraclypeal area wider than torulus. Subantennal groove nearly as long as torulus. 587 Distance from torulus to median ocellus $0.9 \times$ distance from torulus to oral margin. POL $3.1 \times$ 
588 OOL. Scape $2.2-2.5 \times$ as long as pedicel. Antenna with two anelli. First funicular segment 0.7$5890.8 \times$ as long as wide, with $12-15$ multiporous plate sensilla.

590 Mesosoma. Mesoscutum reticulate. Mesoscutum and scutellar-axillar complex not strongly 591 curved in lateral view. Notaulus crenulated. Mesoscutellum 1.3× as long as wide near transscutal 592 articulation. Axillula with longitudinal striae. Frenal sulcus conspicuous, barely crenulated or 593 crenulated. Metascutellum nearly as long as, or longer than frenum in dorsal view. Anterior 594 margin of propodeum angulose medially. Propodeal median line present, traceable at least in the 595 anterior half of sclerite. Stigmal vein $0.9 \times$ as long as marginal vein, with 2 adstigmal setae. 596 Postmarginal vein very short, as long as $1 / 3 \times$ stigmal vein length.

597 Metasoma. Ovipositor sheaths $0.8-0.9 \times$ as long as hind tibia.

598

599 Male. Similar to female. Predominant body colour paler, yellow white. Metasoma dorsally 600 brown, or sometimes completely brown. Distance from torulus to median ocellus $1.3 \times$ distance 601 from torulus to oral margin. POL 2.1× OOL. Mesoscutellum 1.6× as long as wide near 602 transscutal articulation.

603

604 Host plant. Ficus citrifolia Miller.

605

606

607

Idarnes brunneus Farache \& Rasplus, sp. n.

608 (Figs 2F, 4F, 6F, 8F, 10F, 12F, 14F)

609

610 Type material. Holotype: $q$, COSTA RICA: Limón: near Bananito, $9.838917^{\circ}-83.048111^{\circ}$, 611 15.IV.2010, Cruaud A. \& Rasplus J.Y., ex Ficus colubrinae n JRAS02832_05 \& JRAS02833 612 (CBGP).

613

614 Paratypes. COSTA RICA: Limón: 3 km W Guacimo, 10.211873 -83.716842 , 1 \% , 2.III.2008, 615 Rasplus J.Y. \& Ramírez W., ex Ficus colubrinae n JRAS02282_03 (CBGP), near Bananito, $6169.838917^{\circ}-83.048111^{\circ}, 17 q, 7 \widehat{\jmath}, 15 . I V .2010$, Cruaud A. \& Rasplus J.Y., ex Ficus colubrinae $\mathrm{n}^{\mathrm{o}}$

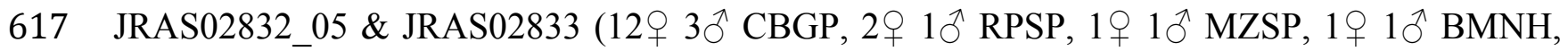
6181 우 $\left.1{ }^{\Uparrow} \mathrm{SAMC}\right)$. 
620 Etymology. The specific name refers to the predominant body colour.

621

622 Diagnosis (+). Body colour predominantly dark brown. Pronotum laterally yellow. Legs yellow, 623 femora and coxae yellow brown. Supraclypeal area as wide as torulus, or slightly narrower. 624 Subantennal groove as long as torulus. Distance from torulus to median ocellus $0.9 \times$ distance 625 from torulus to oral margin. Metascutellum nearly $0.5 \times$ as long as frenum to inconspicuous in 626 dorsal view. Ovipositor sheaths $1.5 \times$ as long as hind tibia.

627

628 Female.

629 Size and colour. Body length 1.3-1.6 mm. Ovipositor length $0.5 \mathrm{~mm}$. Predominantly dark brown. 630 Scape yellow. Pedicel and flagellum yellow brown. Lower face yellow. Pronotum laterally 631 yellow. Legs yellow, femora and coxae yellow brown.

632 Head. Supraclypeal area as wide as torulus. Subantennal groove as long as torulus. Distance 633 from torulus to median ocellus $0.9 \times$ distance from torulus to oral margin. POL $2.9 \times$ OOL. Scape $6342.1 \times$ as long as pedicel. Antenna with two anelli (character sometimes inconspicuous). First 635 funicular segment $0.7-0.8 \times$ as long as wide, with 5-9 multiporous plate sensilla.

636 Mesosoma. Mesoscutum reticulate to punctate reticulate. Mesoscutum and scutellar-axillar 637 complex not strongly curved in lateral view. Notaulus crenulated. Mesoscutellum $1.2 \times$ as long as 638 wide near transscutal articulation. Axillula longitudinally striate to reticulate. Frenal sulcus 639 barely crenulated and inconspicuous. Metascutellum nearly $0.5 \times$ as long as frenum to 640 inconspicuous in dorsal view. Anterior margin of propodeum concave medially. Propodeal 641 median line present as a faint longitudinal reticulation. Stigmal vein $0.9 \times$ as long as marginal 642 vein, with 2-3 adstigmal setae. Postmarginal vein very short, as long as $1 / 3 \times$ stigmal vein length. 643 Metasoma. Ovipositor sheaths $1.5 \times$ as long as hind tibia.

644

645 Male. Similar to female. Funicular segments, pronotum and legs yellow. Distance from torulus 646 to median ocellus $1.2 \times$ distance from torulus to oral margin. POL $2.6 \times$ OOL.

647

648 Host plant. Ficus colubrinae Standley.

649 
650 Idarnes comptoni Farache \& Rasplus, sp. n.

651 urn:lsid:zoobank.org:act:3D938DAE-2869-40B2-888B-041FC96A7FDB

652 (Figs 2G, 4G, 6G, 8G, 10G, 12G, 14G)

653

654 Type material. Holotype: ${ }_{+}$, COSTA RICA: Puntarenas: $8 \mathrm{~km}$ N Ciudad Neily, $8.435083^{\circ}$ $65583.413833^{\circ}$, 23.IV.2010, Cruaud A. \& Rasplus J.Y., ex Ficus hartwegii n JRAS02861 (CBGP). 656

657 Paratypes: COSTA RICA: Puntarenas: 8km N Ciudad Neily, $8.435083^{\circ}-83.413833^{\circ}, 4{ }_{+}, 1{ }^{\lambda}$, 658 23.IV.2010, Cruaud A. \& Rasplus J.Y., ex Ficus hartwegii n ${ }^{\circ}$ JRAS02861 (2 q 1 § CBGP, 2 q 659 RPSP).

660

661 Etymology. The species is dedicated to our friend and colleague, Dr Stephen G. Compton, for 662 his great contribution to the study of fig wasps and figs.

663

664 Diagnosis (†). Body colour mostly brown. Frons more yellow near clypeus. Supraclypeal area 665 as wide as torulus. Flagellum with 2 anelli. Propodeum dorsally yellow. Postmarginal vein nearly 666 absent. Legs predominantly yellow. Axillula longitudinally striate to reticulate.

667

668

\section{Female.}

669

670 Size and colour. Body length 1.1-1.3 mm. Ovipositor length $0.5 \mathrm{~mm}$. Body colour mostly

671 brown. Scape and pedicel yellow. Flagellomeres yellow brown. Frons more yellow near clypeus.

672 Head and mesosoma with faint metallic luster. Tibiae and tarsi yellow.

673 Head. Supraclypeal area as wide as torulus. Subantennal groove as long as torulus. Distance 674 from torulus to median ocellus $1 \times$ distance from torulus to oral margin. POL $2.8 \times$ OOL. Scape $6752 \times$ as long as pedicel. Antenna with two anelli. First funicular segment $0.6 \times$ as long as wide, 676 with 3-4 multiporous plate sensilla.

677 Mesosoma. Mesoscutum reticulate. Mesoscutum and scutellar-axillar complex not strongly 678 curved in lateral view. Notaulus mostly without crenulation. Mesoscutellum $1.1 \times$ as long as wide 679 near transcutal articulation. Axillula longitudinally striate to reticulate. Frenal sulcus barely 680 crenulated, inconspicuous. Metascutellum inconspicuous in dorsal view. Anterior margin of 
681 propodeum concave medially. Propodeal median line present as a faint longitudinal reticulation.

682 Stigmal vein $0.6 \times$ as long as marginal vein, with 3 adstigmal setae. Postmarginal vein nearly

683 absent, shorter than $1 / 5 \times$ stigmal vein length.

684 Metasoma. Ovipositor sheaths $1.7-1.8 \times$ as long as hind tibia.

685

686 Male. Similar to female.

687

688

Host plant. Ficus hartwegii (Miquel) Miquel.

689

690

Idarnes cremersiae Farache \& Rasplus, sp. n.

691 urn:lsid:zoobank.org:act:5F8B227F-7568-4965-BBA4-41C8AEE08EB3

692 (Figs 2H, 4H, 6H, 8H, 10H, 12H, 14H)

693

694 Type material. Holotype: q, FRENCH GUIANA: savanne roche, route de Kourou à 695 Sinnamary, $5.115317^{\circ}-52.783200^{\circ}$, 16.V.2011, Conchou L., ex Ficus cremersii, n ${ }^{\circ}$ JRAS03711 $^{2}$ 696 (CBGP).

697

698 Paratypes: FRENCH GUIANA: savanne roche, route de Kourou à Sinnamary, 5.115317 $69952.783200^{\circ}, 2 \bigcirc, 2 \hat{\jmath}, 16 . V .2011$, Conchou L., ex Ficus cremersii, no JRAS03711 (19 1ð CBGP, $700191 \hat{\jmath}$ RPSP).

701

702 Etymology. The specific name refers to the host plant.

703

704 Diagnosis ( $(+)$. Head yellow, brown in dorsal view. Mesosoma predominantly brown. Pronotum 705 in lateral view and prepectus mostly yellow. Axillula longitudinally striate to reticulate. 706 Ovipositor sheaths $1.5 \times$ as long as hind tibia.

707

708 Female.

709 Size and colour. Body length 1.8-2mm. Ovipositor length $0.6 \mathrm{~mm}$. Head yellow, brown in dorsal 710 view. Scape yellow, pedicel and flagellomeres yellow brown. Mesosoma predominantly brown.

711 Pronotum in lateral view and prepectus mostly yellow. Axillula slightly metallic green. 
712 Propodeum yellow. Legs predominantly yellow, slightly brown. Metasoma brown black.

713 Head. Supraclypeal area as wide as torulus. Subantennal groove as long as torulus. Distance

714 from torulus to median ocellus $1 \times$ distance from torulus to oral margin. POL $2.7 \times$ OOL. Scape

$7152.2 \times$ as long as pedicel. Flagellum with 2 anelli. First funicular segment $0.9 \times$ as long as wide, 716 with 8-11 multiporous plate sensilla.

717 Mesosoma. Mesoscutum reticulate. Mesoscutum and scutellar-axillar complex not strongly 718 curved in lateral view. Notaulus with shallow crenulation. Mesoscutellum $1.2 \times$ as long as wide 719 near transcutal articulation. Axillula longitudinally striate to reticulate. Frenal sulcus barely 720 crenulated, conspicuous. Metascutellum nearly $0.5 \times$ as long as frenum to inconspicuous in dorsal 721 view. Anterior margin of propodeum concave medially. Propodeal median line present, 722 conspicuous. Stigmal vein $0.7 \times$ as long as marginal vein, with 1 adstigmal seta. Postmarginal 723 vein nearly absent, shorter than $1 / 5 \times$ stigmal vein length.

724 Metasoma. Ovipositor sheaths $1.5 \times$ as long as hind tibia.

725

726 Male. Similar to female, body mostly pale yellow and wings medially infuscate. POL 1.7× OOL. 727 Mesoscutellum $1.4 \times$ as long as wide near transcutal articulation.

728

729

Host plant. Ficus cremersii Berg.

730

731

732

Idarnes dimorphicus Farache \& Rasplus, sp. n.

733 (Figs 2I, 4I, 6I, 8I, 10I, 12I, 14I)

734

735 Type material. Holotype: + , BRAZIL: São Paulo: Gália, $-22.30241^{\circ}-49.62102^{\circ}$, 696m, 736 9.VII.2009, Farache F.H.A., ex Ficus citrifolia no FHAF00183_05 (MZSP).

737

738 Paratypes. BRAZIL: Amazonas: Manaus, -3.06º $-60.11^{\circ}, 2$,, 23. VIII.2006, Santos, O.A., ex

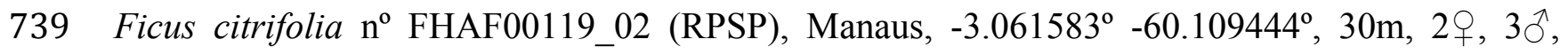
740 6.X.2011, Farache F.H.A. \& Costa P.C., ex Ficus citrifolia n ${ }^{\circ}$ FHAF00235_06 (RPSP), Manaus, 741 Ponta Negra, Hotel Tropical, $-3.06^{\circ}$, $-60.11^{\circ}$, 6q, 2ð, 23.VIII.2006, Santos O.A., ex Ficus

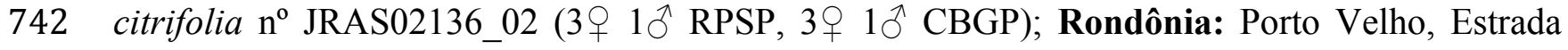


743 Belmont, -8.66937 ${ }^{\circ},-63.91303^{\circ}, 69 \mathrm{~m}, 19 \bigcirc, 3 ð$, 28.VIII.2012, Farache F.H.A. \& Costa P.C., ex

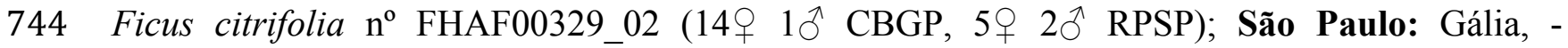
$74522.39544^{\circ},-49.78056^{\circ}, 656 \mathrm{~m}, 5 q, 22 . I X .2008$, Cerezini M.T. \& Farache F.H.A., ex Ficus 746 citrifolia $\mathrm{n}^{\mathrm{o}}$ FHAF00065_07 (RPSP), Gália, -22.3748 ${ }^{\circ},-49.6911^{\circ}, 676 \mathrm{~m}, 5$, 25.IX.2008, 747 Farache F.H.A. \& Pereira R.A.S., ex Ficus citrifolia nº FHAF00064_04 (CBGP), Gália, $74822.30241^{\circ},-49.62102^{\circ}, 696 \mathrm{~m}, 19$, 9へ̂, 9.VII.2009, Farache F.H.A., ex Ficus citrifolia $\mathrm{n}^{\mathrm{o}}$

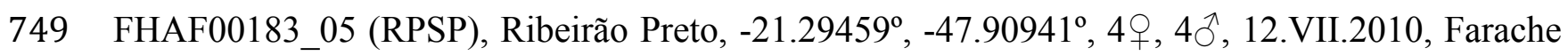

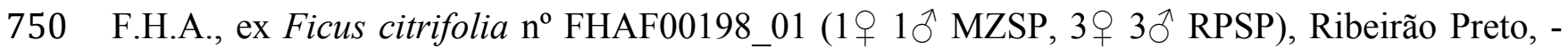
$75121.19216^{\circ},-47.78117^{\circ}, 10 \bigcirc, 1 \hat{\jmath}, 23 . I V .2009$, Cerezini M.T. \& Teixeira L.M.R., ex Ficus

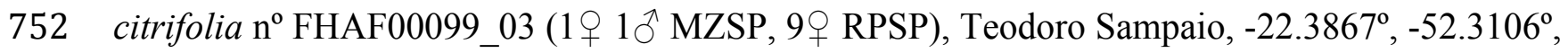

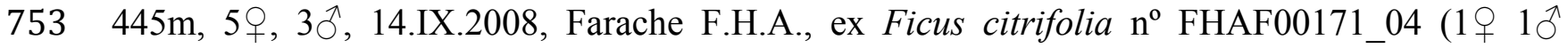
$\left.754 \mathrm{BMNH}, 1 \uparrow 1 \mathrm{O}^{\Uparrow} \mathrm{SAMC}, 1 \uparrow 1 \delta \mathrm{MZSP}, 2 \uparrow \mathrm{RPSP}\right)$.

755

756

Etymology. The name refers to the sexual colour dimorphism observed in this species.

757

758 Diagnosis (ㅇ). Head and mesosoma predominantly yellow orange. Metasoma black.

759 Predominant colour of males brown black. Supraclypeal area as wide as torulus. Subantennal 760 groove longer than torulus. Distance from torulus to median ocellus $1-1.1 \times$ the distance from 761 torulus to oral margin. Metascutellum nearly $0.3-1.0 \times$ as long as frenum in dorsal view. Anterior 762 margin of propodeum angulose medially. Ovipositor sheaths $0.9-1 \times$ as long as hind tibia.

763

\section{Female.}

765 Size and colour. Body length 1.7-2.1 mm. Ovipositor length 0.4-0.5 mm. Head and mesosoma 766 yellow orange. Vertex dark orange, black in ocellar margin. Antennae and legs paler. Metasoma 767 black. Ovipositor sheaths $0.9-1 \times$ as long as hind tibia.

768 Head. Supraclypeal area as wide as torulus. Subantennal groove as long as, or slightly longer 769 than torulus. Distance from torulus to median ocellus $1-1.1 \times$ distance from torulus to oral 770 margin. POL 2.5-3× OOL. Scape $2-2.5 \times$ as long as pedicel. Antenna with two anelli. First 771 funicular segment $0.7-0.9 \times$ as long as wide, with 9-14 multiporous plate sensilla.

772 Mesosoma. Mesoscutum reticulate. Mesoscutum and scutellar-axillar complex not strongly 773 curved in lateral view. Notaulus crenulated. Mesoscutellum 1.2-1.3× as long as wide near 
774 transscutal articulation. Axillula with longitudinal striae. Frenal sulcus barely crenulated and 775 conspicuous. Metascutellum nearly $0.3-1.0 \times$ as long as frenum in dorsal view. Anterior margin 776 of propodeum angulose medially. Propodeal median line present, traceable at least in the anterior 777 half of propodeum. Stigmal vein $0.9 \times$ as long as marginal vein, with $2-3$ adstigmal setae. 778 Postmarginal vein very short, as long as $1 / 3 \times$ stigmal vein length.

779 Metasoma. Ovipositor sheaths $0.9-1 \times$ as long as hind tibia.

780

781 Male. Similar to female. Body predominantly brown black. Legs distally yellow. Antennae 782 yellow brown.

783

784

Host plant. Ficus citrifolia Miller.

785

786

787

Idarnes flavicrus Farache \& Rasplus, sp. n.

788

urn:lsid:zoobank.org:act:4DFA1180-913B-48E1-B228-1ACE7F4603F0

(Figs 3A, 5A, 7A, 9A, 11A, 13A, 15A)

789

790

791

Type material. Holotype: ${ }^{+}$, COSTA RICA: La Fortuna: Arenal, $10.49916^{\circ}-84.71019^{\circ}$,

792

793

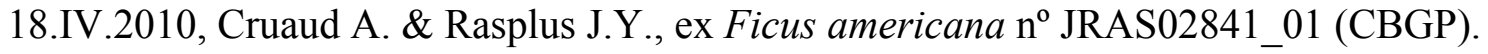

794

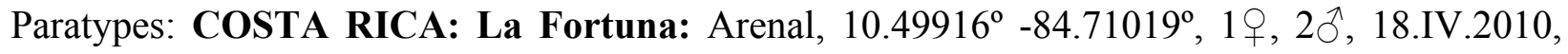
795

796

797

Cruaud A. \& Rasplus J.Y., ex Ficus americana no JRAS02841_01 (1 1 2 20 CBGP).

798

799

Diagnosis ( $९$ ). Head yellow orange. Mesosoma and metasoma predominantly brown black.

800 Supraclypeal area narrower than torulus. Subantennal groove shorter than torulus. Distance from 801 torulus to median ocellus $1-1.2 \times$ distance from torulus to oral margin. Notaulus nearly without 802 crenulation. Axillula reticulate, without longitudinal striae. Frenal sulcus smooth and 803 inconspicuous. Metascutellum inconspicuous in dorsal view. Wing with with 4 adstigmal setae. 804 Ovipositor sheaths $2-2.1 \times$ as long as hind tibia. 
806

807 Sizt

808 809

810

811

812

813

814

815

816

817

818

819

820

821

822

823

824

825

826

827

828

829

830

831

832

833 Type material. Holotype: + , COSTA RICA: Heredia: Santo Domingo, 8.435083 ${ }^{\circ}$ $83483.413833^{\circ}$, 12.IV.2010, Cruaud A. \& Rasplus J.Y., ex Ficus aurea form isophlebia $\mathrm{n}^{\mathrm{o}}$ 835

Size and colour. Body length $1.8 \mathrm{~mm}$. Ovipositor length $0.7-0.8 \mathrm{~mm}$. Head yellow orange. Scape and pedicel yellow. Flagellum yellow brown. Mesosoma bown black. Pronotum laterally yellow. Legs yellow. Metasoma brown black

Head. Supraclypeal area narrower than torulus. Subantennal groove shorter than torulus. Distance from torulus to median ocellus $1-1.2 \times$ distance from torulus to oral margin. POL $2.9 \times$ OOL. Scape $2.3 \times$ as long as pedicel. Antenna with two anelli. First funicular segment $0.8-0.9 \times$ as long as wide, with approximately 6 multiporous plate sensilla.

Mesosoma. Mesoscutum slightly reticulate. Mesoscutum and scutellar-axillar complex not strongly curved in lateral view. Notaulus nearly without crenulation. Mesoscutellum $1.1 \times$ as long as wide near transscutal articulation. Axillula reticulate, without longitudinal striae. Frenal sulcus smooth and inconspicuous. Metascutellum inconspicuous in dorsal view. Anterior margin of propodeum concave medially. Propodeal median line present as a faint longitudinal reticulation. Stigmal vein $0.6 \times$ as long as marginal vein, with 4 adstigmal setae. Postmarginal vein short, as long as $1 / 3 \times$ stigmal vein length.

Metasoma. Ovipositor sheaths $2-2.1 \times$ as long as hind tibia.

Male. Similar to female. Body colour predominantly yellow. Vertex yellow brown. Mesosoma dorsally yellow brown, particularly brown at mesoscutum and scutellar-axillar complex. Metasoma brown black.

Host plant. Ficus americana subsp. americana Aublet.

Idarnes flaviventris Farache \& Rasplus, sp. n.

urn:1sid:zoobank.org:act:8061097A-9783-4A71-8C5B-017C24BD48B5

(Figs 3B, 5B, 7B, 9B, 11B, 13B, 15B)

JRAS02809 (CBGP). 
837 Paratypes. COSTA RICA: Heredia: Santo Domingo, 8.435083 ${ }^{\circ}, 83.413833^{\circ}, 8 q 4 \overbrace{}^{\lambda}$, 838 12.IV.2010, Cruaud A. \& Rasplus J.Y., ex Ficus aurea form isophlebia $n^{\circ}$ JRAS02809 $(5 q 4 \hat{\jmath}$ 839 CBGP, $1 q$ BMNH, $2 q$ MZSP, 19 SAMC); Limón: 12 Km SW Bribri, 9.559778º -82.9135º, 8406 6 3 ${ }^{\lambda}$ 21.IV.2010, Cruaud A. \& Rasplus J.Y., ex. Ficus aurea form isophlebia, n ${ }^{\circ}$ JRAS02829

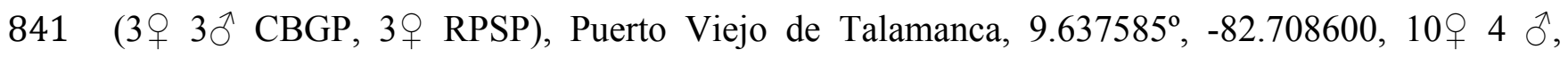
842 14.IV.2010, Cruaud A. \& Rasplus J.Y., ex Ficus aurea form isophlebia n ${ }^{\circ}$ JRAS02824_02 (7q

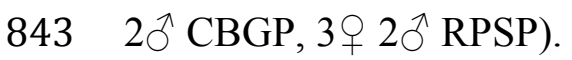

844

845 Etymology. The specific name refers to the coloration of the metasoma, especially in ventral 846 view.

847

848 Diagnosis (q). Body predominantly yellow orange. Margin of metasomal tergites brown. 849 Subantennal groove shorter than torulus. Distance from torulus to median ocellus $1.3 \times$ distance 850 from torulus to oral margin. Metascutellum inconspicuous to approximately $0.5 \times$ as long as 851 frenum in dorsal view. Anterior margin of propodeum angulose medially. Propodeal median line 852 present, traceable at least in the anterior half of propodeum. Ovipositor sheaths $2.7-2.9 \times$ as long 853 as hind tibia.

854

855 Female.

856 Size and colour. Body length 2.2-2.3 mm. Ovipositor length 1.3-1.4 mm. Head, mesosoma, and 857 antennae yellow orange. Legs more yellow. Metasoma yellow, margin of tergites brown.

858 Head. Supraclypeal area narrower than torulus. Subantennal groove shorter than torulus. 859 Distance from torulus to median ocellus $1.3 \times$ distance from torulus to oral margin. POL $2.5 \times$ 860 OOL. Scape $2.2-2.3 \times$ as long as pedicel. Antenna with two anelli (character sometimes 861 inconspicuous). First funicular segment $0.7-0.8 \times$ as long as wide, with $7-8$ multiporous plate 862 sensilla.

863 Mesosoma. Mesoscutum reticulate to punctate reticulate. Mesoscutum and scutellar-axillar 864 complex not strongly curved in lateral view. Notaulus crenulated. Mesoscutellum $1.1 \times$ as long as 865 wide near transscutal articulation. Axillula longitudinally striate to reticulate. Frenal sulcus 866 crenulated and conspicuous. Metascutellum inconspicuous to approximately $0.5 \times$ as long as 
867 frenum in dorsal view. Anterior margin of propodeum angulose medially. Propodeal median line 868 present, traceable at least in the anterior half of propodeum. Stigmal vein $0.7 \times$ as long as 869 marginal vein, with 1-2 adstigmal setae. Postmarginal vein very short, as long as $1 / 3 \times$ stigmal 870 vein length.

871 Metasoma. Ovipositor sheaths 2.7-2.9× as long as hind tibia.

872

873 Male. Similar to female. Distance from torulus to median ocellus $1.7 \times$ distance from torulus to 874 oral margin. POL 2.0× OOL. Antennal flagellum shorter than scape plus pedicel (longer in 875 female).

876

877 Host plant. Ficus aurea Nuttal, form isophlebia (Standley) Berg. Ficus isophlebia was 878 synonymized with $F$. aurea, however the differences observed between entities within the $F$. 879 aurea species complex led C. C. Berg to recognize four informal entities within the species 880 (Berg 2007). Taking into account the morphological differences observed in the host-plants 881 (Ficus aurea form isophlebia and Ficus aurea form aurea), the fact that these forms are 882 sympatrically pollinated by different pollinator species and that non-pollinating communities 883 associated to these forms are composed of different species (JYR, unpublished data), including 884 different species of the Idarnes incertus species-group, we suspect that these forms of Ficus 885 aurea may be in fact different but closely related Ficus species.

886

887

Idarnes gibberosus Farache \& Rasplus, sp. n.

888

urn:1sid:zoobank.org:act:20EC9435-6547-4FFA-AC77-9205B471F40C

889 (Figs 3C, 5C, 7C, 9C, 11C, 13C, 15C)

890

891 Type material. Holotype: + , COLOMBIA: Cundinamarca: Bogota, Ciudad Universitaria, $8924.638568^{\circ}-74.089985^{\circ}, 2620 \mathrm{~m}, 3$. III.2006, Jansen-G. S., ex Ficus americana subsp. andicola $\mathrm{n}^{\mathrm{o}}$ 893 JRAS01682_02 (CBGP).

894

895 Paratype. + , COLOMBIA: Cundinamarca: Bogota, Ciudad Universitaria, 4.638568 $89674.089985^{\circ}, 2620 \mathrm{~m}, 3 . I I I .2006$, Jansen-G. S., ex Ficus americana subsp. andicola $\mathrm{n}^{\mathrm{o}}$ 897 JRAS01682_02 (CBGP). 
898

899 Etymology. The specific name refers to the mesoscutum, which is particularly curved in lateral 900 view.

901

902 Diagnosis (). Body predominantly black. Supraclypeal area narrower than torulus. Subantennal 903 groove longer than torulus. Distance from torulus to median ocellus $0.8 \times$ distance from torulus to 904 oral margin. Mesoscutum and scutellar-axillar complex strongly curved in lateral view. 905 Metascutellum nearly as long as, or longer than frenum in dorsal view. Ovipositor sheaths $2 \times$ as 906 long as hind tibia.

907

908 Female.

909 Size and colour. Body length $1.8 \mathrm{~mm}$. Ovipositor length $0.9 \mathrm{~mm}$. Predominantly black. Scape 910 brown, pedicel and flagellum yellow. Legs brown black. Tibia, tarsi, proximal portion of femur, 911 trochanter and trochantellus yellow.

912 Head. Supraclypeal area narrower than torulus. Subantennal groove longer than torulus. Distance 913 from torulus to median ocellus $0.8 \times$ distance from torulus to oral margin. POL $2.5 \times$ OOL. Scape $9142.3 \times$ as long as pedicel. Antenna with two anelli. First funicular segment $0.7-0.8 \times$ as long as 915 wide, with 8-9 multiporous plate sensilla.

916 Mesosoma. Mesoscutum reticulate to punctate reticulate. Mesoscutum and scutellar-axillar 917 complex strongly curved in lateral view. Notaulus sparsely crenulated. Mesoscutellum $1.2 \times$ as 918 long as wide near transscutal articulation. Axillula longitudinally striate to reticulate. Frenal 919 sulcus crenulated and conspicuous. Metascutellum nearly as long as, or longer than frenum in 920 dorsal view. Anterior margin of propodeum angulose medially. Propodeal median line present, 921 conspicuous. Stigmal vein as long as 0.6× marginal vein, with 2 adstigmal setae. Postmarginal 922 vein nearly as long as $0.5 \times$ stigmal vein length.

923 Metasoma. Ovipositor sheaths $2 \times$ as long as hind tibia.

924

925 Male. Not known.

926

927 Host plant. Ficus americana subsp. andicola (Standley) Berg. 928 
929 Idarnes gordhi Farache \& Rasplus, sp. n.

930 urn:lsid:zoobank.org:act:57B16D6D-A205-4F3A-B53E-E58399809FEC

931 (Figs 3D, 5D, 7D, 9D, 11D, 13D, 15D)

932

933 Type material. Holotype: + , COSTA RICA: Limón: $8 \mathrm{Km}$ W Guapiles $10.20650^{\circ}-83.86173^{\circ}$, 934 13.IV.2010, ex. Ficus popenoei, no JRAS02812_2, Cruaud A. \& Rasplus, J.Y. leg. (CBGP).

935 Paratypes: COSTA RICA: Limón: $8 \mathrm{Km} \mathrm{N}$ Gualipes, $10.20650^{\circ}-83.86173^{\circ}$, 5q, $4 \overbrace{}^{\lambda}$, 936 13.IV.2010, Cruaud A. \& Rasplus J.Y., ex Ficus popenoei n ${ }^{\circ}$ JRAS02812_02 (3q 3ð CBGP, $1 q$ $9371 \delta$ RPSP, 1 \% MZSP).

938

939 Etymology. The species is dedicated to the renowned entomologist, Gordon Gordh.

940

941 Diagnosis (†). Head and mesosoma yellow orange. Metasoma brown black. Supraclypeal area 942 slightly wider than torulus. Subantennal groove shorter than torulus. Distance from torulus to 943 median ocellus 1.0-1.1× distance from torulus to oral margin. Metascutellum inconspicuous in 944 dorsal view. Ovipositor sheaths $2.4-2.5 \times$ as long as hind tibia.

945

946 Female.

947 Size and colour. Body length $2 \mathrm{~mm}$. Ovipositor length 1.1-1.2 mm. Head and mesosoma yellow 948 orange. Antennae and legs yellow orange. Lateral panel of metanotum brown black. Propodeum 949 more yellow. Metasoma brown black.

950 Head. Supraclypeal area slightly wider than torulus. Subantennal groove shorter than torulus. 951 Distance from torulus to median ocellus $1.1 \times$ distance from torulus to oral margin. POL $2.5 \times$ 952 OOL. Scape $2.2 \times$ as long as pedicel. Antenna with two anelli (character sometimes 953 inconspicuous). First funicular segment $0.8 \times$ as long as wide, with 6-8 multiporous plate sensilla. 954 Mesosoma. Mesoscutum reticulate. Mesoscutum and scutellar-axillar complex not strongly 955 curved in lateral view. Notaulus crenulated, crenulation very shallow. Mesoscutellum $1.4 \times$ as 956 long as wide near transscutal articulation. Axillula reticulate. Frenal sulcus barely crenulated and 957 inconspicuous. Metascutellum inconspicuous in dorsal view. Anterior margin of propodeum 958 concave medially. Propodeal median line present, traceable at least in the anterior half of 
959 propodeum. Stigmal vein as long as marginal vein, with 2-4 adstigmal setae. Postmarginal vein

960 nearly absent, shorter than $1 / 5 \times$ stigmal vein length.

961 Metasoma. Ovipositor sheaths 2.4-2.5× as long as hind tibia.

962

963 Male. Similar to female. Body colour paler. Distance from torulus to median ocellus $1.7 \times$ 964 distance from torulus to oral margin. POL $1.9 \times$ OOL. First funicular segment $0.5 \times$ as long as 965 wide.

966

967 Host plant. Ficus popenoei Standley.

968

969

Idarnes hansoni Bouček, 1993

970 (Figs 3E, 5E, 7E, 9E, 11E, 13E, 15E)

971

9721993 Bouček, Z., Journal of Natural History 27: 202-203, Fig. 38. Description (†

973

974 Type material. Holotype: + , COSTA RICA: San José: Zarcero, Llano Bonito, XII.1987, 975 Hanson P., ex Ficus (BMNH, examined).

976

977 Paratypes. COSTA RICA: Guanacaste: N.P. Santa Rosa, 1 9 , I.1987, Gauld, I (BMNH); San 978 José: Zarcero, Llano Bonito, 4, XII.1987, Hanson P., ex Ficus (BMNH), Zurqui de Moravia, 979 1600m, 1, 2ð, 7-9.IX.1991, Hanson P., ex Ficus brenesii (=F. hartwegii) (EBCR, USNM, 980 $\mathrm{BMNH})$

981

982 Diagnosis (). Body predominantly brown black. Supraclypeal area wider than torulus. 983 Subantennal groove as long as torulus. Distance from torulus to median ocellus $0.9 \times$ distance 984 from torulus to oral margin. Metascutellum inconspicuous in dorsal view. Anterior margin of 985 propodeum concave medially. Ovipositor sheaths $1.4-1.5 \times$ as long as hind tibia.

986

987 Description: See supplementary material 5

988

989 Host plant. Ficus hartwegii (Miquel) Miquel. Ficus brenesii Standl. is considered a junior 
990 synonym of $F$. hartwegii (Miq.).

991

992 Remarks. One paratype analysed (Guanacaste, N. P. Santa Rosa, January 1987, I. Gauld leg. $993(\mathrm{BMNH}))$ actually belongs to an undescribed species. Since only one specimen is known and 994 because we have no host information, we decided not to describe it waiting for more information 995 and specimens. This species can be distinguished from I. hansoni by the following characters: (1) 996 head, pronotum, and propodeum yellow brown, (2) propodeal median line present and 997 conspicuous, (3) anterior margin of propodeum slightly angulose medially.

998

999

Idarnes incertus (Ashmead, 1900)

1000

(Figs 3F, 5F, 7F, 9F, 11F, 13F, 15F)

1001

1002

1900 Ashmead, W.H., Transactions of the Entomological Society of London 33:253 Description 1003 († Љ̂) (Comb.: Sycophila incerta).

1004

1005

1993 Bouček, Z., Journal of Natural History 27: 202, Fig. 37. Lectotype designation. (Comb.: 1006 Idarnes incerta).

1007

1008

Type material. Lectotype. $q$, USA: Florida: Coconut Grove (USNM).

1009 Paralectotypes: ST. VINCENT: 2q, Smith H.H. (USNM). USA: Florida: Florida city, $1 \hat{\jmath}$, 1010 V.1989, Nadel H., ex Ficus citrifolia (BMNH)

1011

1012 Diagnosis ( $(+)$. Body predominantly yellow orange. Metasoma dorsally brown black, first tergite 1013 yellow. Supraclypeal area as wide as torulus. Subantennal groove as long as torulus. Distance 1014 from torulus to median ocellus $1 \times$ distance from torulus to oral margin. Frenal sulcus smooth. 1015 Metascutellum nearly $0.5 \times$ as long as frenum to inconspicuous in dorsal view. Postmarginal vein 1016 nearly absent, shorter than $1 / 5 \times$ stigmal vein length. Ovipositor sheaths $1.4 \times$ as long as hind tibia. 1017

1018 Description: See supplementary material 5

1019

1020

Host plant. Ficus aurea form aurea Nuttal and Ficus citrifolia Miller 
1021

1022 Remarks. Several specimens collected in Guadeloupe (38, 7ð̋, JRAS01219 \& JRAS01220, 1023 CBGP, RPSP) are probably closely related to Idarnes incertus, yet, subtle morphological 1024 differences can be observed between these specimens and the type specimens from Florida. 1025 Consequently, Idarnes incertus may constitute a complex of species associated with Ficus aurea 1026 and F. citrifolia in Florida and in the Caribbean islands. Therefore this species may deserve 1027 thorough phylogeographical analyses using large sampling before a better species delimitation.

1028

1029

Idarnes maximus Farache \& Rasplus, sp. n.

1030 urn:1sid:zoobank.org:act:BA9EEC28-FD78-45B6-953A-11274C64995E

1031 (Figs 3G, 5G, 7G, 9G, 11G, 13G, 15G)

1032

1033 Type material. Holotype: + , BRAZIL: São Paulo: Gália, -22.2949º, -49.64812 ${ }^{\circ}$, 31.III.2008, 1034 Farache F.H.A., ex Ficus obtusifolia n ${ }^{\circ}$ FHAF00015_02 (MZSP).

1035

1036

Paratypes. BRAZIL: São Paulo: Araraquara, Road to Fazenda Salto Grande, $-21.804685^{\circ}$ 1037 48.203512 $2^{\circ}$ 634m, 7, 7ð,, 30.VII.2012, Farache F.H.A., ex Ficus obtusifolia $\mathrm{n}^{\mathrm{o}}$ 1038

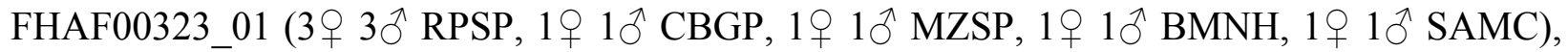
1039

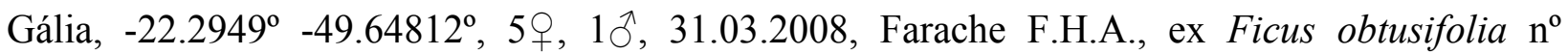

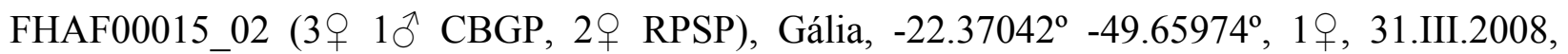

1041 Farache F.H.A., ex Ficus obtusifolia n n FHAF00011_12 (RPSP), Gália, -22.37852 ${ }^{\circ}-49.71912^{\circ}$, 10423 39, 9.VII.2009, Teixeira L.M.R. \& Medeiros M.D.F., ex Ficus obtusifolia n ${ }^{\circ}$ FHAF00155_17

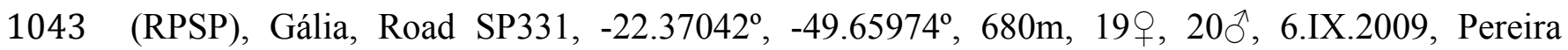
1044 R.A.S., ex Ficus obtusifolia no FHAF00201_05 (RPSP), Garça, -22.2916 -49.74199º 666m, 1045 24ㅇ, 5ภ, 20.XI.2008, Teixeira L.M.R., ex Ficus obtusifolia n ${ }^{\circ}$ FHAF00070_04 (RPSP), 1046 Ribeirão Preto, Bosque Municipal Fábio Barreto, -21.1734 47.8018², 550m, 19, 2.VII.2006, 1047 Farache F.H.A. \& do Ó V.T., ex Ficus obtusifolia no FHAF00134_03 (RPSP).

1048

1049 Etymology. The specific name refers to the large body size of this species. 
1051 Diagnosis (). Head and mesosoma predominantly yellow orange in lateral view. Mesonotum 1052 and lateral panel of metascutum predominantly black in dorsal view. Metasoma brown black. 1053 Supraclypeal area narrower than torulus. Subantennal groove shorter than torulus. Distance from 1054 torulus to median ocellus $1.2 \times$ distance from torulus to oral margin. First funicular segment with 1055 12-17 multiporous plate sensilla. Ovipositor sheaths $2.7-2.8 \times$ as long as hind tibia.

1056

\section{Female.}

1058 Size and colour. Body length 2.4-3.0 mm. Ovipositor length 1.4-1.6 mm. Head and mesosoma 1059 predominantly yellow orange in lateral view. Vertex brown, black near ocelli. Antenna yellow. 1060 Pronotum slightly brown. Mesonotum and lateral panel of metascutum predominantly black in 1061 dorsal view. Legs yellow orange. Mesepimeron black. Mesepisternum ventrally brown black. 1062 Metasoma brown black.

1063 Head. Supraclypeal area narrower than torulus. Subantennal groove shorter than torulus. 1064 Distance from torulus to median ocellus $1.2 \times$ distance from torulus to oral margin. POL $2.2 \times$ 1065 OOL. Scape $2.3 \times$ as long as pedicel. Antenna with two anelli. First funicular segment $0.9 \times$ as 1066 long as wide, with 12-17 multiporous plate sensilla.

1067 Mesosoma. Mesoscutum reticulate. Mesoscutum and scutellar-axillar complex not strongly 1068 curved in lateral view. Notaulus crenulated. Mesoscutellum 1.1-1.2× as long as wide near 1069 transscutal articulation. Axillula with longitudinal striae. Frenal sulcus barely crenulated and 1070 faint. Metascutellum nearly $0.5-1.0 \times$ as long as frenum in dorsal view. Anterior margin of 1071 propodeum angulose medially. Propodeal median line present, traceable at least in the anterior 1072 half of propodeum. Stigmal vein $0.8 \times$ as long as marginal vein, with 2 adstigmal setae. 1073 Postmarginal vein as long as $0.33-0.5 \times$ stigmal vein length.

1074 Metasoma. Ovipositor sheaths $2.7-2.8 \times$ as long as hind tibia.

1075

1076 Male. Similar to female. Head yellow brown to brown, especially at frons and near vertex in 1077 some specimens. Distance from torulus to median ocellus $1.3-1.4 \times$ distance from torulus to oral 1078 margin. POL $1.8 \times$ OOL.

1079

1080 Host plant. Ficus obtusifolia Kunth 
1082 Idarnes nigriventris Farache \& Rasplus, sp. n.

1083 urn:1sid:zoobank.org:act:0BE190F5-9E67-45CE-9533-30F65905294E

1084 (Figs 3H, 5H, 7H, 9H, 11H, 13H, 15H)

1085

1086

Type material. Holotype: + , COSTA RICA: Heredia: Santo Domingo, $8.435083^{\circ}-83.413833^{\circ}$, 1087

12.IV.2010, Cruaud A. \& Rasplus J.Y., ex Ficus aurea form isophlebia nº JRAS02809 (CBGP).

1088

1089

Paratypes. COSTA RICA: Heredia: Santo Domingo, $8.435083^{\circ}-83.413833^{\circ}, 3 q 1 \delta^{\lambda}$, 1090 12.IV.2010, Cruaud A. \& Rasplus J.Y., ex Ficus aurea form isophlebia nº JRAS02809 (CBGP); 1091

Limón: 12 Km SW Bribri, 9.559778 -82.9135², 6ㅇ 2ð 21.IV.2010, Cruaud A. \& Rasplus J.Y., 1093

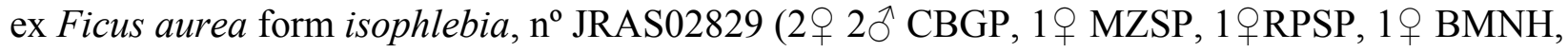
$1 \uparrow$ SAMC), Puerto Viejo de Talamanca, 9.637565 -82.708577, $3 \uparrow$ 2へ̂, 14.IV.2010, Cruaud A. 1095 \& Rasplus J.Y., ex Ficus aurea form isophlebia n $n^{0}$ JRAS02824_02 (2q 1ð CBGP, 1910

Etymology. The specific name refers to the colouration of the metasoma.

1098

Diagnosis ( $(+)$. Head and mesosoma predominantly yellow orange. Metasoma brown black, first tergite yellow. Supraclypeal area narrower than torulus. Subantennal groove shorter than torulus. Distance from torulus to median ocellus $0.9 \times$ distance from torulus to oral margin. Metascutellum nearly $0.5 \times$ as long as frenum or shorter in dorsal view. Ovipositor sheaths 1.9$2.2 \times$ as long as hind tibia.

1104

1105

Female.

1106 Size and colour. Body length 1.7-2.1 mm. Ovipositor length 0.8-0.9 mm. Head and mesosoma 1107 yellow orange. Antennae and legs paler. Metasoma brown black, first tergite yellow.

1108 Head. Supraclypeal area narrower than torulus. Subantennal groove shorter than torulus. 1109 Distance from torulus to median ocellus $0.9 \times$ distance from torulus to oral margin. POL $2.8 \times$ 1110 OOL. Scape $2.3 \times$ as long as pedicel. Antenna with two anelli. First funicular segment $0.7 \times$ as 1111 long as wide, with 5-7 multiporous plate sensilla. 
1112 Mesosoma. Mesoscutum reticulate to punctate reticulate. Mesoscutum and scutellar-axillar 1113 complex not strongly curved in lateral view. Notaulus with shallow crenulation. Mesoscutellum

$11141.1 \times$ as long as wide near transscutal articulation. Axillula with longitudinal striae. Frenal sulcus 1115 crenulated and conspicuous. Metascutellum nearly $0.5 \times$ as long as frenum or shorter in dorsal 1116 view. Anterior margin of propodeum angulose medially. Propodeal median line present, 1117 traceable at least in the anterior half of propodeum. Stigmal vein $0.9 \times$ as long as marginal vein, 1118 with 2 adstigmal setae. Postmarginal vein very short, as long as $1 / 3 \times$ stigmal vein length.

1119 Metasoma. Ovipositor sheaths 1.9-2.2× as long as hind tibia.

1120

1121 Male. Similar to female. Distance from torulus to median ocellus $1.2 \times$ distance from torulus to 1122 oral margin. POL $2.4 \times$ OOL.

1123

1124 Host plant. Ficus aurea Nuttal form isophlebia (Standley) Berg.

1125

1126

Idarnes pseudoflavus Farache \& Rasplus, sp. n.

1127 urn:lsid:zoobank.org:act:5E9EB253-0BCD-4EA4-A788-0FEED5DE482D

1128 (Figs 3I, 5I, 7I, 9I, 11I, 13I, 15I)

1129

1130

1131

Type material. Holotype: ${ }^{+}$, COSTA RICA: San José: Pérez Zeledón, $9.337597^{\circ}-83.641458^{\circ}$, 1132

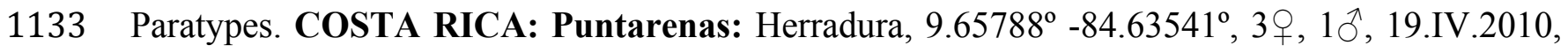
1134 Cruaud A. \& Rasplus J.Y., ex Ficus goldmanii (=F. crocata) no JRAS02843_01 (CBGP); San 1135 José: Pérez Zeledón, 9.337597 -83.641458, 68ㅇ, 17ð, 26.II.2008, Rasplus J.Y., ex Ficus 1136

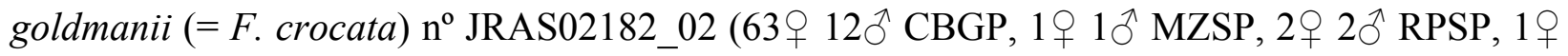
$11371 ð \mathrm{BMNH}, 1$ 우 $1 \precsim \mathrm{SAMC})$.

1138

1139 Etymology. The specific name refers to the predominant body colour.

1140

1141 Diagnosis. Head and mesosoma predominantly yellow orange. Metasoma yellow brown to 1142 black. Supraclypeal area narrower than torulus. Subantennal groove shorter than torulus. 
1143 Distance from torulus to median ocellus $1.4 \times$ distance from torulus to oral margin.

1144 Metascutellum inconspicuous in dorsal view. Ovipositor sheaths $1.8 \times$ as long as hind tibia.

1145

1146 Female.

1147 Size and colour. Body length 2-2.3 mm. Ovipositor length 0.8-0.9 mm. Head, mesosoma, 1148 antennae and legs predominantly yellow orange. Pronotum laterally yellow. Lateral panel of 1149 metanotum brown. Propodeum yellow. Mesepisternum, mesepimeron and mesocoxa slightly 1150 brown. Metasoma dorsally brown black, laterally yellow, and ventrally brown-yellow.

1151 Head. Supraclypeal area narrower than torulus. Subantennal groove shorter than torulus. 1152 Distance from torulus to median ocellus $1.4 \times$ distance from torulus to oral margin. POL $2.9 \times$ 1153 OOL. Scape 2.3-2.6× as long as pedicel. Antenna with one anellus. First funicular segment 0.6$11540.8 \times$ as long as wide, with 7-13 multiporous plate sensilla.

1155 Mesosoma. Mesoscutum reticulate. Mesoscutum and scutellar-axillar complex not strongly 1156 curved in lateral view. Notaulus crenulated. Mesoscutellum 1.3× as long as wide near transscutal 1157 articulation. Axillula with longitudinal striae. Frenal sulcus crenulated. Metascutellum 1158 inconspicuous in dorsal view. Anterior margin of propodeum angulose medially. Propodeal 1159 median line present, traceable at least in the anterior half of propodeum. Stigmal vein $0.9 \times$ as 1160 long as marginal vein, with 2 adstigmal setae. Postmarginal vein very short, as long as $1 / 3 \times$ 1161 stigmal vein length.

1162 Metasoma. Ovipositor sheaths $1.8 \times$ as long as hind tibia.

1163

1164 Male. Similar to female. Predominant body colour is paler, yellow white. Metasoma brown 1165 black except the first and second tergites, which are yellow. Distance from torulus to median 1166 ocellus $2.1 \times$ distance from torulus to oral margin. POL $2.2 \times$ OOL

1167

1168 Host plant. Ficus crocata (Miquel) Miquel.

1169

1170

Idarnes ramirezi Farache \& Rasplus, sp. n.

1171 urn:lsid:zoobank.org:act:470B1ABF-F6C6-4C8B-BBE5-69ABD5548EA8

1172 (Figs 3J, 5J, 7J, 9J, 11J, 13J, 15J)

1173 
1174 Type material. Holotype: + , COSTA RICA: Guanacaste: Pequeña Helvetia, Hotel de los 1175 Heroes, $10.475466^{\circ}-84.830086^{\circ}$, 5.III.2008, Rasplus J.Y. \& Ramírez W., ex Ficus perforata $(=$ 1176 F. americana subsp. americana) no JRAS02177_03 (CBGP).

1178 Paratypes: COSTA RICA: Guanacaste: Pequeña Helvetia, Hotel de los Heroes, $10.475466^{\circ}$ $117984.830086^{\circ}, 11$, $15 \AA$, 5.III.2008, Rasplus J.Y. \& Ramírez W., ex Ficus perforata $(=F$. 1180 americana subsp. americana) $\mathrm{n}^{\circ}$ JRAS02177_03 (6우 10へ CBGP, 2 우 $2 \hat{0} \mathrm{RPSP}, 1$ 우 $1 \hat{0} \mathrm{MZSP}$,

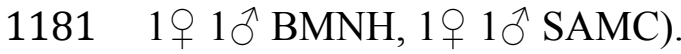

1182

1183 Etymology. The specific name is dedicated to our friend and colleague, Dr. William Ramírez, 1184 for his great contribution to the study of fig wasps and figs. The specimens belonging to this 1185 species were collected thanks to his valuable help and deep knowledge of the figs of Costa Rica.

1186

1187 Diagnosis (ㅇ). Head and mesosoma yellow brown. Mesoscutellum, frenum and axillula smoky 1188 yellow to brown. Propodeum yellow. Metasoma brown black. Supraclypeal area wider than 1189 torulus. Subantennal groove as long as, or slightly longer than torulus. Distance from torulus to 1190 median ocellus $0.9 \mathrm{x}$ distance from torulus to oral margin. Metascutellum inconspicuous in dorsal 1191 view. Ovipositor sheaths $1.3-1.4 \times$ as long as hind tibia.

1192

1193 Female.

1194 Size and colour. Body length 1.7-1.8 mm. Ovipositor length $0.5 \mathrm{~mm}$. Head and mesosoma 1195 yellow brown. Antennae and legs yellow orange. Vertex slightly brown. Mesoscutellum, frenum 1196 and axillula slightly brown. Propodeum yellow. Metasoma brown black.

1197 Head. Supraclypeal area wider than torulus. Subantennal groove as long as, or slightly longer 1198 than torulus. Distance from torulus to median ocellus $0.9 \times$ distance from torulus to oral margin. 1199 POL $3 \times$ OOL. Scape $1.8-2.2 \times$ as long as pedicel. Antenna with two anelli. First funicular 1200 segment $0.6-0.7 \times$ as long as wide, with 5-8 multiporous plate sensilla.

1201 Mesosoma. Mesoscutum reticulate to punctate reticulate. Mesoscutum and scutellar-axillar 1202 complex not strongly curved in lateral view. Notaulus crenulated. Mesoscutellum $1.2 \times$ as long as 1203 wide near transscutal articulation. Axillula longitudinally striate to reticulate. Frenal sulcus 1204 barely crenulated and inconspicuous. Metascutellum inconspicuous in dorsal view. Anterior 
1205 margin of propodeum concave medially. Propodeal median line present as a faint longitudinal 1206 reticulation. Stigmal vein $0.8 \times$ as long as marginal vein, with 3 adstigmal setae. Postmarginal 1207 vein nearly absent, shorter than $1 / 5 \times$ stigmal vein length.

1208 Metasoma. Ovipositor sheaths $1.3-1.4 \times$ as long as hind tibia.

1209

1210 Male. Similar to female. Mesosoma paler. Mesoscutellum, frenum, axillula and propodeum 1211 yellow. Head smoky yellow to brown. Distance from torulus to median ocellus $1.4 \times$ distance 1212 from torulus to oral margin. POL $2.1 \times$ OOL .

1213

1214 Host plant. Ficus americana subsp. americana Aublet.

1216

1217

1218

1219

1220

1221

1222

1223

1224

1225

1226

1227

1228

1229

1230

1231

1232

1233

1234

1235

Phylogenetic analyses

Our alignment consisted of 4024 bp $(C O I=1466$ bp; $C y t B=712$ bp; EF-1 $\alpha=517$ bp; $28 S \operatorname{rRNA}=1329 \mathrm{bp}$ ). Protein translations revealed no stop codons or frame shifts. Models chosen by AIC for each partition were GTR + $\Gamma(m t D N A \& 28 S r R N A)$, and $\mathrm{K} 80+\Gamma(E F-1 \alpha)$.

The trees reconstructed using ML and Bayesian methods showed the same topology (Fig. 16). Idarnes incertus species-group was recovered monophyletic $\left(\mathrm{PP}=1 ; \mathrm{ML}_{\mathrm{BP}}=100 \%\right)$ and divided in two main clades (clade 1 and clade 2; Fig. 16). The first clade is well resolved and composed by five species; I. brunneus was recovered sister to I. comptoni $\left(\mathrm{PP}=1 ; \mathrm{ML}_{\mathrm{BP}}=\right.$ $100 \%$ ), and I. amazonicus was sister to I. gordhi plus I. ramirezi ( $\left.\mathrm{PP}=1 ; \mathrm{ML}_{\mathrm{BP}}=100 \%\right)$. The deeper nodes within the second clade were not well resolved, yet we could retrieve a well supported clade formed by I. pseudoflavus, I. brevis, and I. dimorphicus $\left(\mathrm{PP}=1 ; \mathrm{ML}_{\mathrm{BP}}=100 \%\right)$ but the relationships among these tree species were uncertain. Also, I. incertus was retrieved as sister to I. flaviventris + I. nigriventris $\left(\mathrm{PP}=1 ; \mathrm{ML}_{\mathrm{BP}}=100 \%\right)$. The relationships of I. maximus and I. gibberosus were not well established (Fig. 16). Idarnes maximus was recovered sister to $I$. brevis + I. dimorphicus + I. pseudoflavus with relatively high Bayesian posterior probability support $(\mathrm{PP}=0.97)$ but low maximum likelihood booststrap support $\left(\mathrm{ML}_{\mathrm{BP}} 52 \%\right)$, while $I$. gibberosus was recovered as sister to the clade I. flaviventris $+I$. incertus + I. nigriventris with

Bayesian posterior probability support $(\mathrm{PP}=0.9)$ yet low $\mathrm{ML}_{\mathrm{BP}} \operatorname{support}\left(\mathrm{ML}_{\mathrm{BP}}=63 \%\right)$

\section{Discussion}


1236

1237

1238

1239

1240

1241

1242

1243

1244

1245

1246

1247

1248

1249

1250

1251

1252

1253

1254

1255

1256

1257

1258

1259

1260

1261

1262

1263

1264

1265

1266

The Idarnes incertus species-group is clearly distinct from the remaining Idarnes. Indeed, they rarely exhibit metallic tinge and their ovipositor sheaths are always shorter than the body length, whereas the remaining Idarnes exhibit metallic colour and the ovipositor is always longer than body. Species belonging to the I. incertus species-group are globally similar, and the main differences between species concern the body coloration and the relative length of the ovipositor. According to recent phylogenetic analyses, the I. incertus species-group appears to be a recent radiation within Sycophaginae (Cruaud et al. 2011a; Cruaud et al. 2011b) and the morphological similarity of the species may be partly linked to their recent divergence ( $20-10 \mathrm{Ma}$, during the Miocene; Cruaud et al. 2011a).

Species of the Idarnes incertus species-group are usually species-specific with the exception of $I$. americanae, that was found associated with $F$. americana and $F$. costaricana (both species occurring in Costa Rica), and I. incertus that is associated with F. aurea and $F$. citrifolia in Florida.

Several fig species host more than one species of the I. incertus species-group:

1) Four species are associated with Ficus americana, namely I. americanae, I. flavicrus, I. gibberosus, and I. ramirezi. The former two species occurred together within figs of $F$. americana subsp. americana in Costa Rica, while I. ramirezi occurred in the same subspecies, but in different samples. Idarnes gibberosus occurred in figs of F. americana subsp. andicola in Colombia. Ficus aurea hosted three species; the co-occurring I. flaviventris and I. nigriventris in Costa Rica and I. incertus in Florida.

2) Ficus citrifolia hosts different species in different parts of its distribution range: $I$. dimorphicus occurs in South America (Brazil: Amazonas, Rondônia, and São Paulo), while I. brevis occurs in F. citrifolia in Costa Rica, and I. incertus in Florida.

3) Ficus hartwegii is the host plant of I. comptoni and I. hansoni in Costa Rica.

4) Finally, Ficus pertusa hosts I. amacayacuensis and I. badiovertex.

These patterns strongly suggest that the diversification of the I. incertus species-group within Ficus do not follow a "one-to-one rule" of diversification as discussed for pollinators 
1267 (Rasplus 1996). Our results clearly show that host shifts between Ficus species and 1268 diversification on the same Ficus host are frequent. Our phylogenetic analyses show one case of 1269 diversification within the same host species. Indeed, species associated with the Ficus aurea 1270 complex (I. flaviventris + I. incertus + I. nigriventris) formed a strongly supported monophyletic 1271 clade and were morphologically closely related. On the other hand, species associated with the $F$. 1272 americana complex (I. amazonicus, I. gibberosus, and I. ramirezi) belong to different clades, 1273 which strongly suggests that host shifts happened. Patterns of diversification within host species 1274 were also observed in Anidarnes for which sister species occurred on the same host complexes, 1275 i.e. F. aurea and F. americana complexes (Farache et al. 2013). This suggests the existence of 1276 different diversification patterns among genera even when they show similar life histories and 1277 belong to a same subfamily.

1278 Here we recognized three previously described species belonging to Idarnes incertus 1279 species-group. Additionally, 17 species new to science are recognized and described. Most 1280 species were collected in Costa Rica (13 species), and some from Brazil (4 species) and 1281 Colombia (3 species). Sampling efforts in Brazil and Costa Rica are comparable, and therefore 1282 this shows that the group is probably more diversified in lower latitudes. The high number of 1283 new species found in this study highlights the lack of taxonomic information on the Neotropical 1284 fig wasps. Despite an increasing number of phylogenetic studies including these wasps (Cruaud 1285 et al. 2011a; Cruaud et al. 2010; Cruaud et al. 2011b; Cruaud et al. 2012; Heraty et al. 2013; 1286 Munro et al. 2011) just a few recent (i.e. 20th century onwards) taxonomic papers are available 1287 on non-pollinating and pollinating wasps (Bouček 1993; Farache et al. 2013; Jansen-Gonzalez \& 1288 Sarmiento 2008; Schiffler et al. 2002; Wiebes 1995).

1289 This study yields taxonomic and phylogenetic frameworks for a group of Idarnes, which 1290 represents an important part of the Sycophaginae diversity (ca. 33\% of the species). This 1291 contribution is an important step to a well-established taxonomic foundation for Agaonidae, and 1292 we hope it will subsidize further investigations addressing taxonomy, evolution, and host 1293 specificity in fig wasps.

1294

\section{Acknowledgements}

1296 We are much indebted to Michael Gates (USNM \& USDA, Washington DC, USA), 1297 Suzanne Ryder and Natalie Dale-Skey Papilloud (BMNH, London, U.K.), and Dominique 
1298 Zimmermann (NMW, Vienna, Austria) for the loan of type materials. We acknowledge P.

1299 Hanson and W. Ramirez for their kind help in Costa Rica. We also thank Sergio Jansen-G and

1300 Otilene Santos Mattos for contributing with samples. We wish to thank Rodrigo Silva and

1301 Laurent Soldati for help in SEM imaging. We are indebted to Simon van Noort for the assistance

1302 in the deployment of Lucid keys.

1303

1304

1305

1306

1307

1308

1309

1310

1311

1312

1313

1314

1315

1316

1317

1318

1319

1320

1321

1322

1323

1324

1325

1326

1327

1328

1329

1330

1331

\section{References}

Akaike H. 1973. Information theory and an extension of the maximum likelihood principle. In: Petrov PN, and Csaki F, editors. Second international symposium on information theory. Budapest: Academiai Kiado. p 267-281.

Arnett RH, Samuelson GA, and Nishida GM. 1993. The Insect and Spider Collections of the World. Gainesville, FL: Sandhill Crane Press, Inc.

Ashmead WH. 1900. Report upon the aculeate Hymenoptera of the islands of St. Vincent and Grenada, with additions to the parasitic Hymenoptera and a list of the described Hymenoptera of the West Indies. Transactions of the Entomological Society of London 33:207-367.

Ashmead WH. 1904. Classification of the Chalcid flies or the superfamily Chalcidioidea, with descriptions of new species in the Carnegie Museum collected in S. America by Herbert H. Smith. Memoirs of the Carnegie Museum 1:225-551.

Berg CC. 2007. Proposals for treating four species complexes in Ficus subgenus Urostigma section Americanae (Moraceae). Blumea 52:295-312. 10.3767/000651907X609034

Bouček Z. 1988. Australasian Chalcidoidea (Hymenoptera). A biosystematic revision of genera of fourteen families, with a reclassification of species: C.A.B. International, Wallingford.

Bouček Z. 1993. The genera of chalcidoid wasps from Ficus fruit in the New World. Journal of Natural History 27:173-217. 10.1080/00222939300770071

Cook JM, and Rasplus J-Y. 2003. Mutualists with attitude: coevolving fig wasps and figs. Trends in Ecology and Evolution 18:241-248. 10.1016/s0169-5347(03)00062-4

Cook JM, and Segar ST. 2010. Speciation in fig wasps. Ecological Entomology 35:54-66. 10.1111/j.1365-2311.2009.01148.x

Cruaud A, Jabbour-Zahab R, Genson G, Couloux A, Peng Y-Q, Rong YD, Ubaidillah R, Pereira RAS, Kjellberg F, van Noort S, Kerdelhue C, and Rasplus J-Y. 2011a. Out of Australia and back again: the world-wide historical biogeography of non-pollinating fig wasps (Hymenoptera: Sycophaginae). Journal of Biogeography 38:209-225. 10.1111/j.13652699.2010.02429.x 
1332 Cruaud A, Jabbour-Zahab R, Genson G, Cruaud C, Couloux A, Kjellberg F, van Noort S, and

1333

1334

1335

1336

1337

1338

1339

1340

1341

1342

1343

1344

1345

1346

1347

1348

1349

1350

1351

1352

1353

1354

1355

1356

1357

1358

1359

1360

1361

1362

1363

1364

1365

1366

1367

1368

Rasplus J-Y. 2010. Laying the foundations for a new classification of Agaonidae (Hymenoptera: Chalcidoidea), a multilocus phylogenetic approach. Cladistics 26:359387. 10.1111/j.1096-0031.2009.00291.x

Cruaud A, Jabbour-Zahab R, Genson G, Kjellberg F, Kobmoo N, van Noort S, Yang D-R, Peng Y-Q, Ubaidillah R, Hanson PE, Santos-Mattos O, Farache FHA, Pereira RAS, Kerdelhue C, and Rasplus J-Y. 2011b. Phylogeny and evolution of life-history strategies in the Sycophaginae non-pollinating fig wasps (Hymenoptera, Chalcidoidea). $B M C$ Evolutionary Biology 11:178. 10.1186/1471-2148-11-178

Cruaud A, Rønsted N, Chantarasuwan B, Chou L-S, Clement WL, Couloux A, Cousins B, Genson G, Harrison RD, Hanson PE, Hossaert-McKey M, Jabbour-Zahab R, Jousselin E, Kerdelhué C, Kjellberg F, Lopez-Vaamonde C, Peebles J, Peng Y-Q, Pereira RAS, Schramm T, Ubaidillah R, van Noort S, Weiblen GD, Yang D-R, Yodpinyanee A, Libeskind-Hadas R, Cook JM, Rasplus J-Y, and Savolainen V. 2012. An extreme case of plant-insect co-diversification: figs and fig-pollinating wasps. Systematic Biology 61:1029-1047. 10.1093/sysbio/sys068

Darriba D, Taboada GL, Doallo R, and Posada D. 2012. jModelTest 2: more models, new heuristics and parallel computing. Nature Methods 9:772-772. 10.1038/nmeth.2109

Elias LG, Menezes Jr AO, and Pereira RAS. 2008. Colonization sequence of non-pollinating fig wasps associated with Ficus citrifolia in Brazil. Symbiosis 45:107-111.

Elias LG, Teixeira SP, Kjellberg F, and Pereira RAS. 2012. Diversification in the use of resources by Idarnes species: bypassing functional constraints in the fig-fig wasp interaction. Biological Journal of the Linnean Society 106:114-122. 10.1111/j.10958312.2012.01851.x

Farache FHA, Cruaud A, Genson G, Pereira RAS, and Rasplus J-Y. 2013. Taxonomic revision and molecular phylogeny of the fig wasp genus Anidarnes Bouček, 1993 (Hymenoptera, Sycophaginae). Systematic Entomology 38:14-34. 10.1111/j.1365-3113.2012.00644.x

Farache FHA, and Rasplus J-Y. 2014. Revision of the Australasian genus Pseudidarnes Girault, 1927 (Hymenoptera, Agaonidae, Sycophaginae). ZooKeys 404:31-70. 10.3897/zookeys.404.7204

Farache FHA, and Rasplus J-Y. 2015. Conidarnes, a new oriental genus of Sycophaginae (Hymenoptera, Agaonidae) associated with Ficus section Conosycea (Moraceae). ZooKeys 539:119-145. 10.3897/zookeys.539.6529

Farache FHA, Rasplus JY, Azar D, and Compton SG. 2016. First record of a non-pollinating fig wasp (Hymenoptera: Sycophaginae) from Dominican amber, with estimation of the size of its host figs. Journal of Natural History 50:2237-2247. $10.1080 / 00222933.2016 .1193646$ 
1369 Galil J, Dulberger R, and Rosen DE. 1970. The effects of Sycophaga sycomori L. on the

1370

1371

1372

1373

1374

1375

1376

1377

1378

1379

1380

1381

1382

1383

1384

1385

1386

1387

1388

1389

1390

1391

1392

1393

1394

1395

1396

1397

1398

1399

1400

1401

1402

1403

1404 structure and development of the syconia of Ficus sycomorus L. New Phytologist 69:103111.

Galil J, and Eisikowitch D. 1969. Further studies on the pollination ecology of Ficus sycomorus L. (Hymenoptera, Chalcidoidea, Agaonidae). Tijdschrift voor Entomologie 112:1-13.

Gibson GAP. 1997. Morphology and terminology. In: Gibson GAP, Huber JT, and Woolley JB, eds. Annotated Keys to the Genera of Nearctic Chalcidoidea (Hymenoptera). Ottawa: National Research Council Press, 16-44.

Gordh G. 1975. The comparative external morphology and systematics of the Neotropical parasitic fig wasp genus Idarnes (Hymenoptera: Torymidae). University of Kansas Science Bulletin 50:389-455.

Gordh G, and Hall JC. 1979. A critical point drier used as a method of mounting insects from alcohol. Entomological News 90:57-59.

Guindon S, and Gascuel O. 2003. A simple, fast, and accurate algorithm to estimate large phylogenies by maximum likelihood. Systematic Biology 52:696-704. $10.1080 / 10635150390235520$

Heraty JM, Burks RA, Cruaud A, Gibson GAP, Liljeblad J, Munro JB, Rasplus JY, Delvare G, Jansta P, Gumovsky AV, Huber JT, Woolley JB, Krogmann L, Heydon S, Polaszek A, Schmidt S, Darling DC, Gates MW, Mottern J, Murray E, DalMolin A, Triapitsyn SV, Baur H, Pinto JD, van Noort S, and Yoder MJ. 2013. A phylogenetic analysis of the megadiverse Chalcidoidea (Hymenoptera). Cladistics 29:466-542. 10.1111/cla.12006

Heraty JM, and Hawks D. 1998. Hexamethyldisilazane - A chemical alternative for drying insects. Entomological News 109:369-374.

Jansen-Gonzalez S, and Sarmiento CE. 2008. A new species of high mountain Andean fig wasp (Hymenoptera: Agaonidae) with a detailed description of its life cycle. Symbiosis 45:135141.

Katoh K, and Standley DM. 2013. MAFFT multiple sequence alignment software version 7: improvements in performance and usability. Molecular Biology and Evolution 30:772780. Doi 10.1093/Molbev/Mst010

Kumar S, Nei M, Dudley J, and Tamura K. 2008. MEGA: a biologist-centric software for evolutionary analysis of DNA and protein sequences. Briefings in Bioinformatics 9:299306. 10.1093/bib/bbn017

Mayr G. 1906. Neue Feigen-Insekten. Entomologische Zeitung Wien 25:153-187.

Miller MA, Pfeiffer W, and Schwartz T. 2010. Creating the CIPRES Science Gateway for inference of large phylogenetic trees. Proceedings of the Gateway Computing Environments Workshop (GCE). New Orleans LA. p 1-8. 
1405

1406

1407

1408

1409

1410

1411

1412

1413

1414

1415

1416

1417

1418

1419

1420

1421

1422

1423

1424

1425

1426

1427

1428

1429

1430

1431

1432

1433

1434

1435

1436

1437

1438

1439

1440

Munro JB, Heraty JM, Burks RA, Hawks D, Mottern J, Cruaud A, Rasplus J-Y, and Jansta P. 2011. A molecular phylogeny of the Chalcidoidea (Hymenoptera). PloS one 6:e27023. 10.1371/journal.pone.0027023

Noyes JS. 1982. Collecting and preserving chalcid wasps (Hymenoptera: Chalcidoidea). Journal of Natural History 16:315-334. 10.1080/00222938200770261

Pereira RAS, Teixeira SP, and Kjellberg F. 2007. An inquiline fig wasp using seeds as a resource for small male production: a potential first step for the evolution of new feeding habits? Biological Journal of the Linnean Society 92:9-17. 10.1111/j.1095-8312.2007.00826.x

Rambaut A, Suchard MA, Xie D, and Drummond AJ. 2013. Tracer v. 1.5. http://beast.bio.ed.ac.uk/Tracer.

Rasplus J-Y. 1996. The one-to-one species-specificity of the Ficus-Agaoninae mutualism : how casual? In: van der Maesen LJG, van der Burgt XM, and van Medenbach de Rooy JM, eds. The Biodiversity of African plants. Wageningen: Kluwer Academic, 639-649.

Rasplus J-Y, Kerdelhue C, Le Clainche I, and Mondor G. 1998. Molecular phylogeny of fig wasps. Agaonidae are not monophyletic. Comptes Rendus de l'Academie des Sciences Serie III Sciences de la Vie 321:517-527. 10.1016/s0764-4469(98)80784-1

Rasplus J-Y, and Soldati L. 2005. Familia Agaonidae. In: Fernández F, and Sharkey MJ, eds. Introdución a los Hymenoptera de la Región Neotropical. Bogotá: Sociedad Colombiana de Entomología \& Universidad Nacional de Colombia, 683-698.

Ronquist F, Teslenko M, van der Mark P, Ayres DL, Darling A, Höhna S, Larget B, Liu L, Suchard MA, and Huelsenbeck JP. 2012. MrBayes 3.2: Efficient Bayesian Phylogenetic Inference and Model Choice across a Large Model Space. Systematic Biology 61:539-542. $10.1093 /$ sysbio/sys029

Schiffler G, Azevedo CO, and Kawada R. 2002. Agaonidae (Hymenoptera, Chalcidoidea) associated to syconium of Ficus clusiifolia (Moraceae) from Tres Praias, Guarapari, Espirito Santo, Brazil. Boletim do Museu de Biologia Mello Leitão 14:5-12.

Stamatakis A. 2014. RAxML version 8: a tool for phylogenetic analysis and post-analysis of large phylogenies. Bioinformatics 30:1312-1313. 10.1093/bioinformatics/btu033

Tzeng H-Y, Tseng L-J, Ou C-H, Lu K-C, Lu F-Y, and Chou L-S. 2008. Confirmation of the parasitoid feeding habit in Sycoscapter, and their impact on pollinator abundance in Ficus formosana. Symbiosis 45:129-134.

van Noort S, and Rasplus J-Y. 2016. figweb. Available at http://www.figweb.org (accessed July 18 2016).

Wang R, Matthews A, Ratcliffe J, Barwell LJ, Peng YQ, Chou LS, Yu H, Yang HW, and Compton SG. 2014. First record of an apparently rare fig wasp feeding strategy: obligate seed predation. Ecological Entomology 39:492-500. 10.1111/een.12122 
1441 Westwood JO. 1840. On caprification as practised upon the Figs in the south of Europe and the

1442

1443

1444

1445 Wiebes JT. 1966. Provisional host catalogue of fig wasps (Hymenoptera, Chalcidoidea). 1446

1447 1448

1449 Levant, with descriptions of the insects employed for that purpose; and observations upon the Agaon paradoxum Dalman. Transactions of the Entomological Society of London 2:214-224.

Zoologische Verhandelingen 83:1-44.

Wiebes JT. 1995. The New World Agaoninae (pollinators of figs). Verhandelingen der Koninklijke Nederlandse Akademie van Wetenschappen Afdeling Natuurkunde Tweede Reeks 94:1-60. 


\section{Figures}

1452

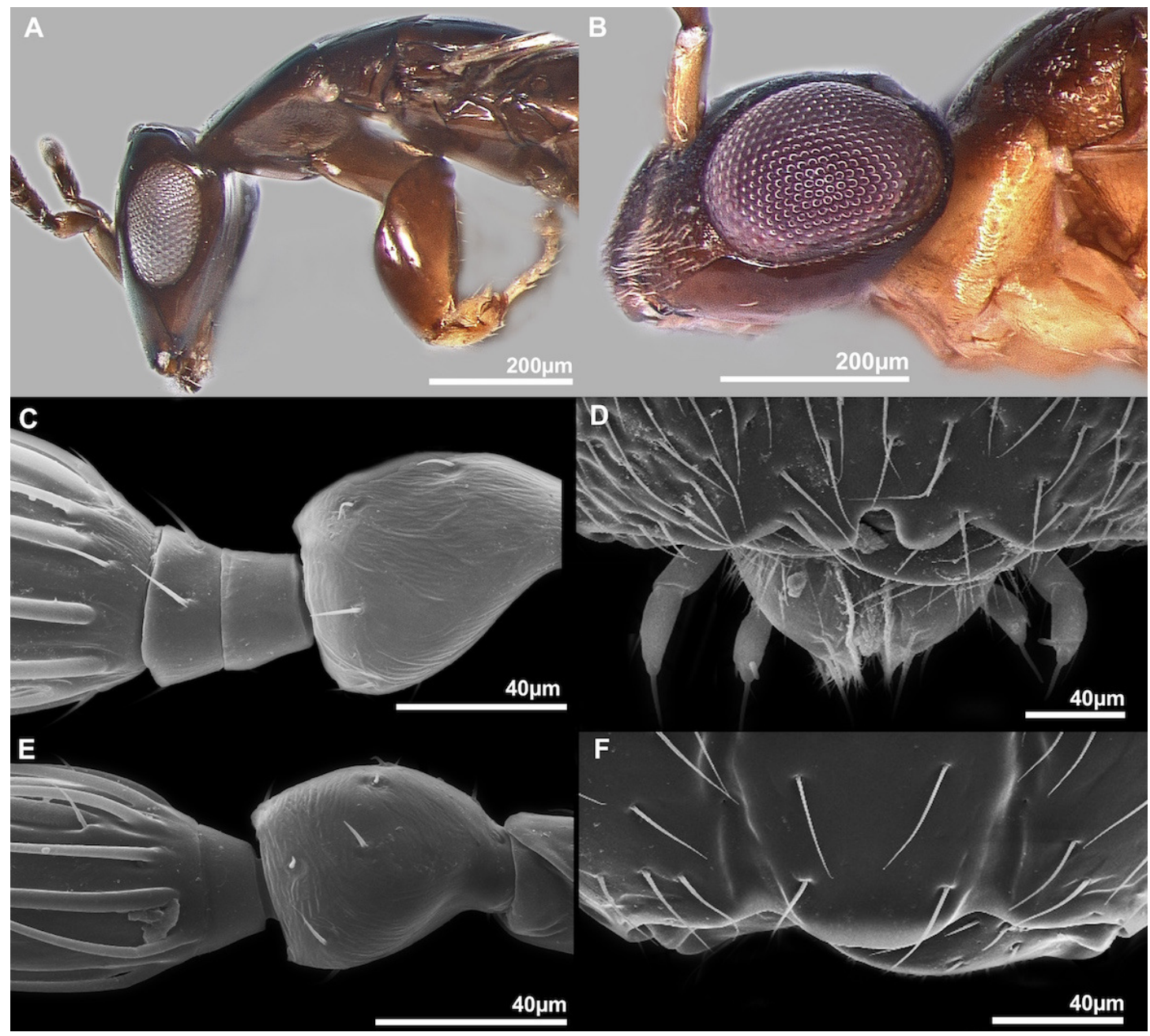

Fig. 1: Sycophaginae morphology. A, Sycophaga sycomori, lateral view of head and mesosoma;

1455 B, Sycophaga testacea, lateral view of head; C, Idarnes flavicollis sp. group, detail of antenna; D,

1456 Idarnes flavicollis sp. group, detail of clypeus; E, Idarnes sp. carme sp. group, detail of antenna;

1457 F, Idarnes sp. carme sp. group, detail of clypeus. 


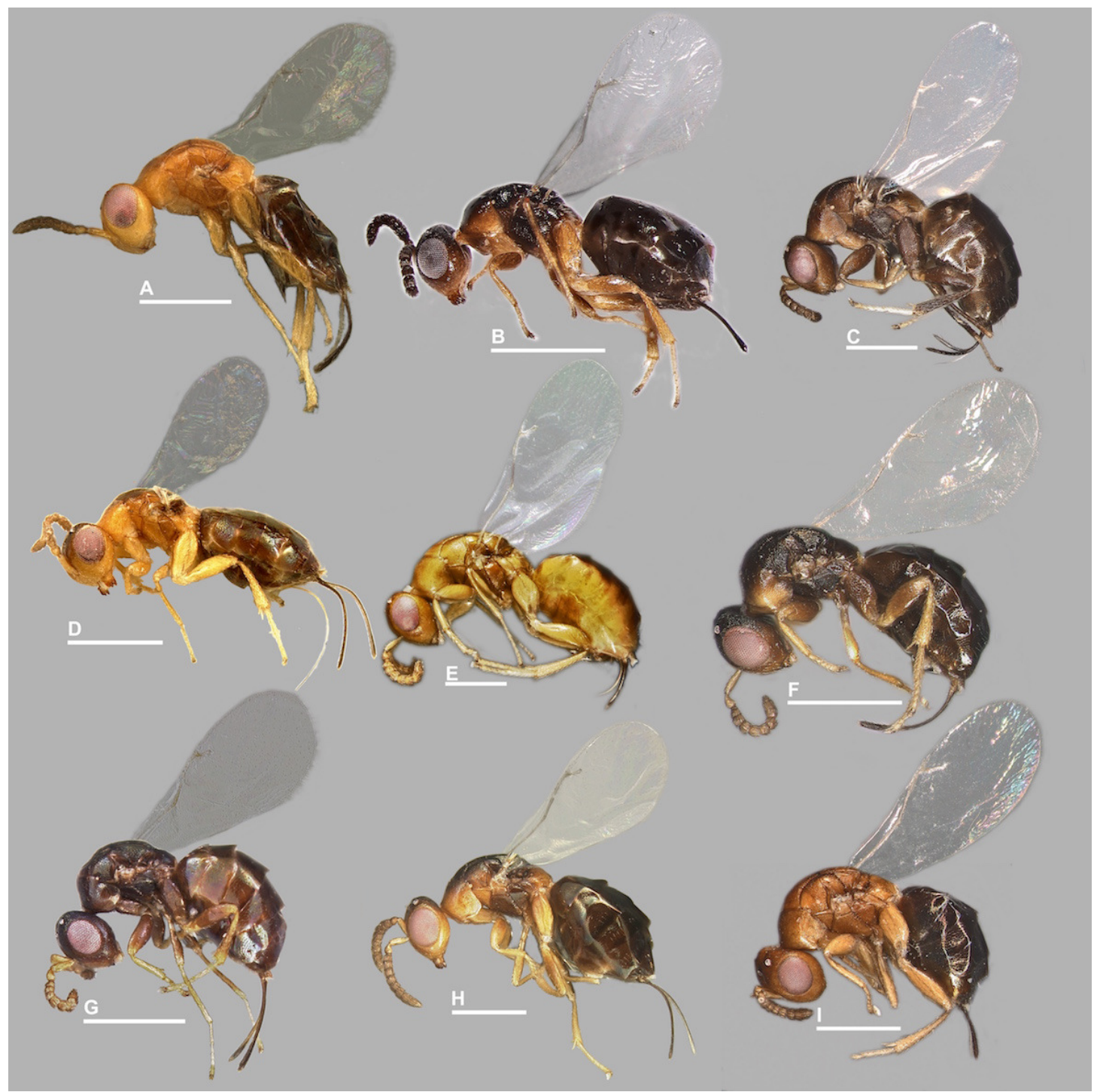

1459 Fig. 2: Habitus in lateral view, Idarnes incertus sp. group, females. A, I. amacayacuensis sp. n.; 1460 B, I. amazonicus sp. n.; C, I. americanae sp. n.; D, I. badiovertex sp. n.; E, I. brevis sp. n.; F, I. 1461 brunneus sp. n.; G, I. comptoni sp. n.; H, I. cremersiae sp. n.; I, I. dimorphicus sp. n. Scale $=500$ $1462 \mu \mathrm{m}$. 


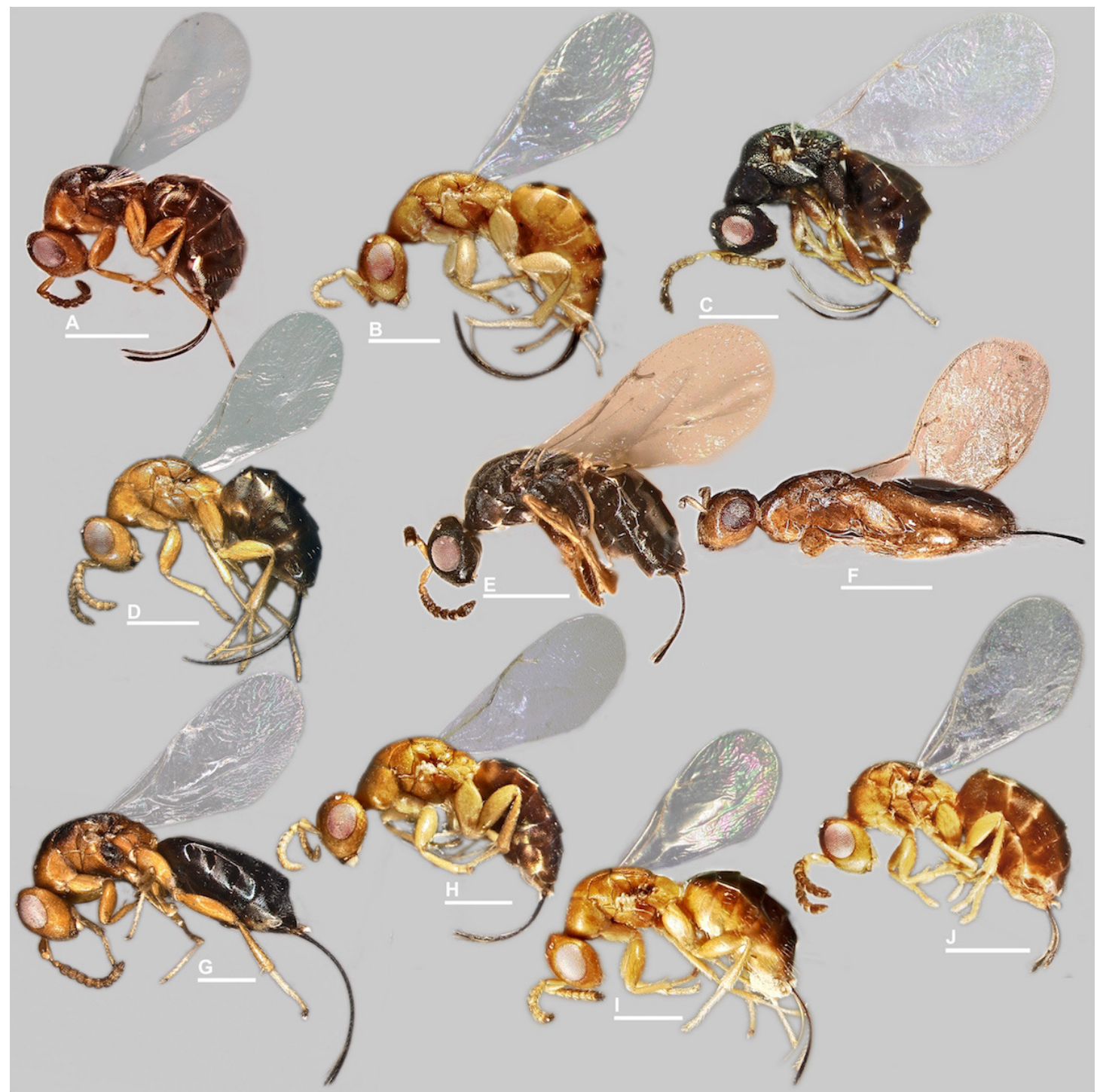

1464 Fig. 3: Habitus in lateral view, Idarnes incertus sp. group, females. A, I. flavicrus sp. n.; B, I. 1465 flaviventris sp. n.; C, I. gibberosus sp. n.; D, I. gordhi sp. n.; E, I. hansoni Bouček, 1993, 1466 Paratype; F, I. incertus Ashmead, 1900, Paralectotype USNM; G, I. maximus sp. n.; H, I. 1467 nigriventris sp. n.; I, I. pseudoflavus sp. n.; J, I. ramirezi sp. n.Scale $=500 \mu \mathrm{m}$. 


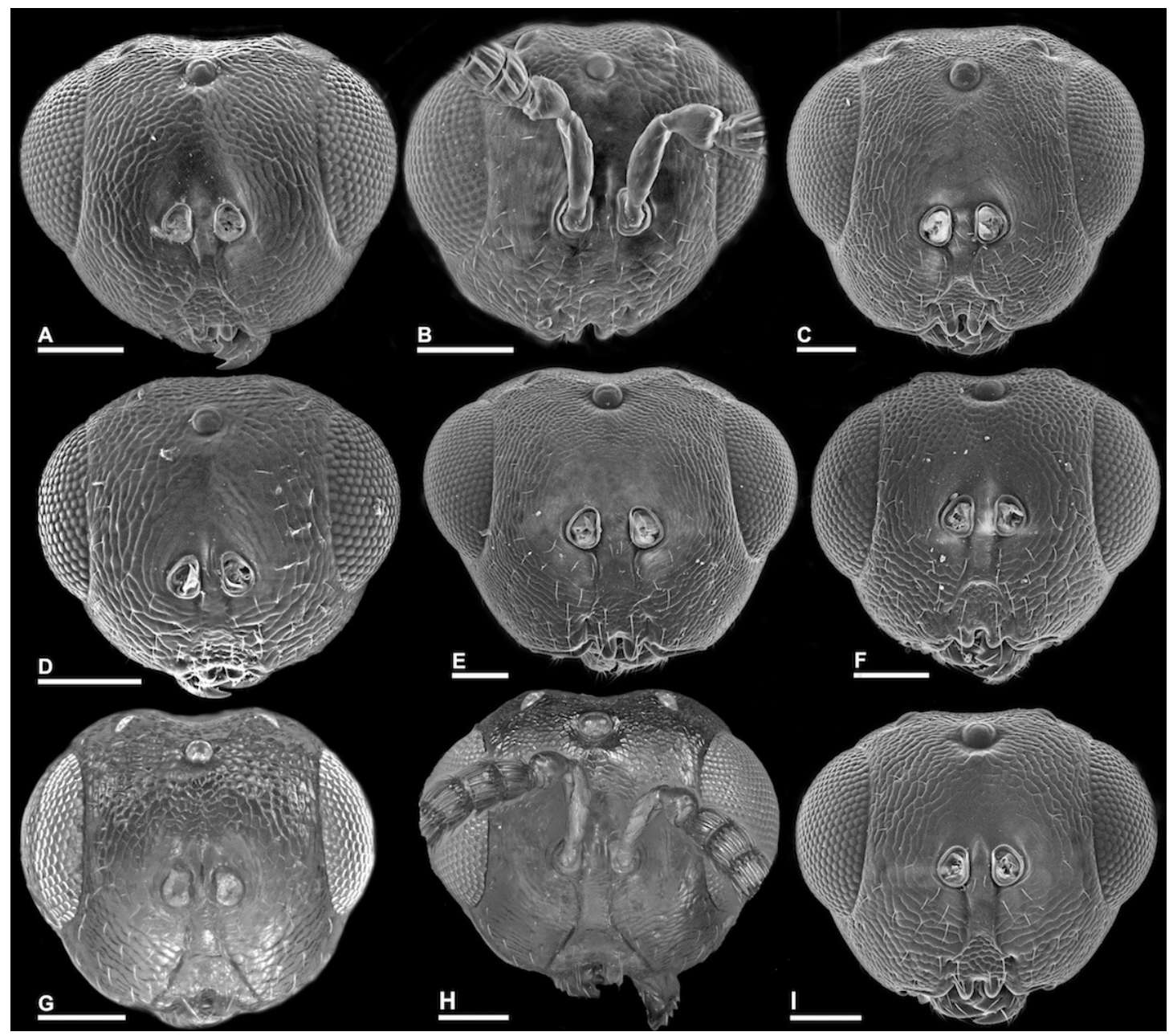

Fig. 4: Head in frontal view, Idarnes incertus sp. group, females. A, I. amacayacuensis sp. n.; B, 1470 I. amazonicus sp. n.; C, I. americanae sp. n.; D, I. badiovertex sp. n.; E, I. brevis sp. n.; F, I. 1471 brunneus sp. n.; G, I. comptoni sp. n.; H, I. cremersiae sp. n.; I, I. dimorphicus sp. n. Scale = $1472100 \mu \mathrm{m}$. 


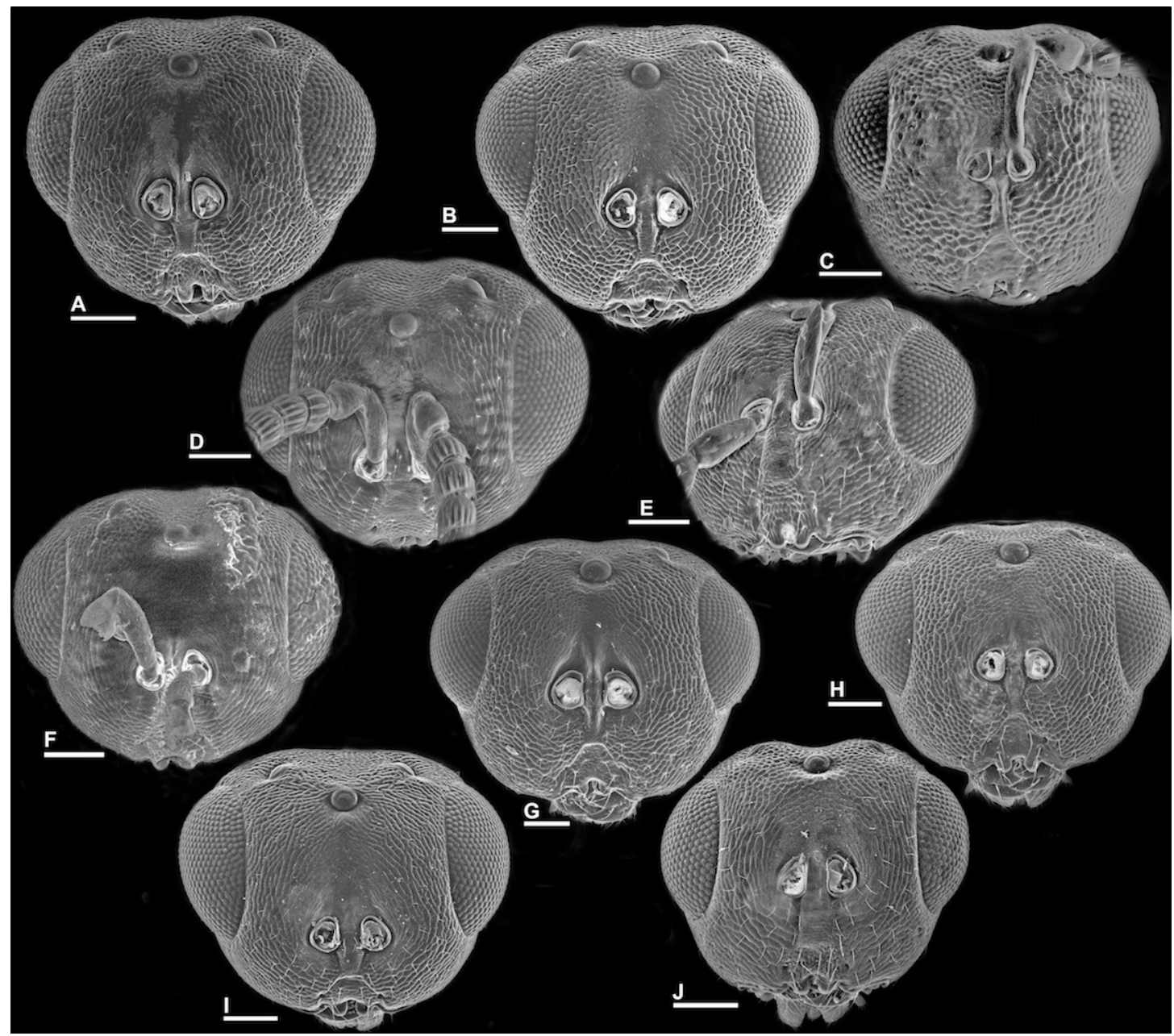

1474 Fig. 5: Head in frontal view, Idarnes incertus sp. group, females. A, I. flavicrus sp. n.; B, I. 1475 flaviventris sp. n.; C, I. gibberosus sp. n.; D, I. gordhi sp. n.; E, I. hansoni Bouček, 1993, 1476 Paratype; F, I. incertus Ashmead, 1900, Paralectotype USNM; G, I. maximus sp. n.; H, I. 1477 nigriventris sp. n.; I, I. pseudoflavus sp. n.; J, I. ramirezi sp. n. Scale $=100 \mu \mathrm{m}$. 


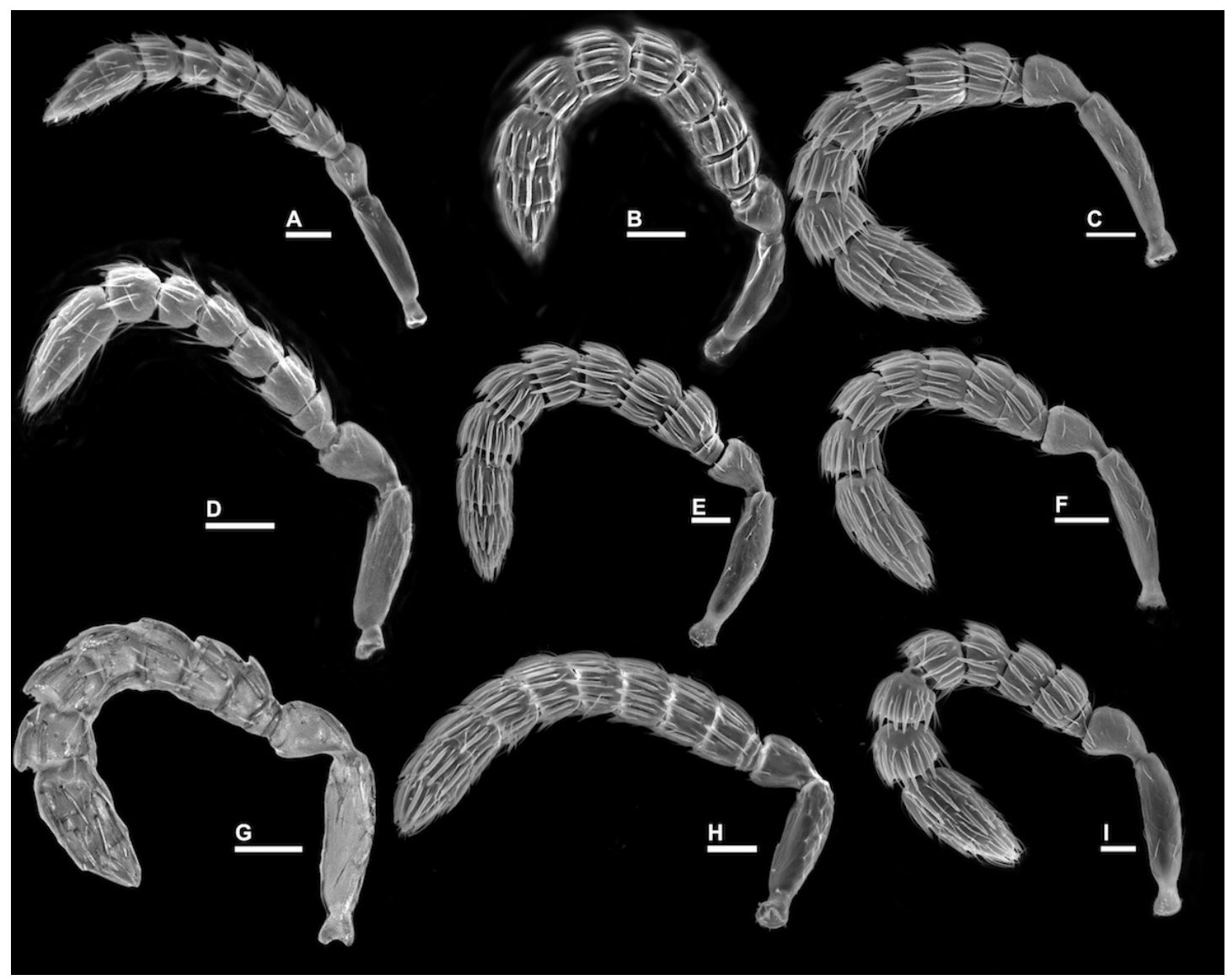

1479 Fig. 6: Antenna, Idarnes incertus sp. group, females. A, I. amacayacuensis sp. n.; B, I. 1480 amazonicus sp. n.; C, I. americanae sp. n.; D, I. badiovertex sp. n.; E, I. brevis sp. n.; F, I. 1481 brunneus sp. n.; G, I. comptoni sp. n.; H, I. cremersiae sp. n.; I, I. dimorphicus sp. n. Scale $=50$ $1482 \mu \mathrm{m}$. 


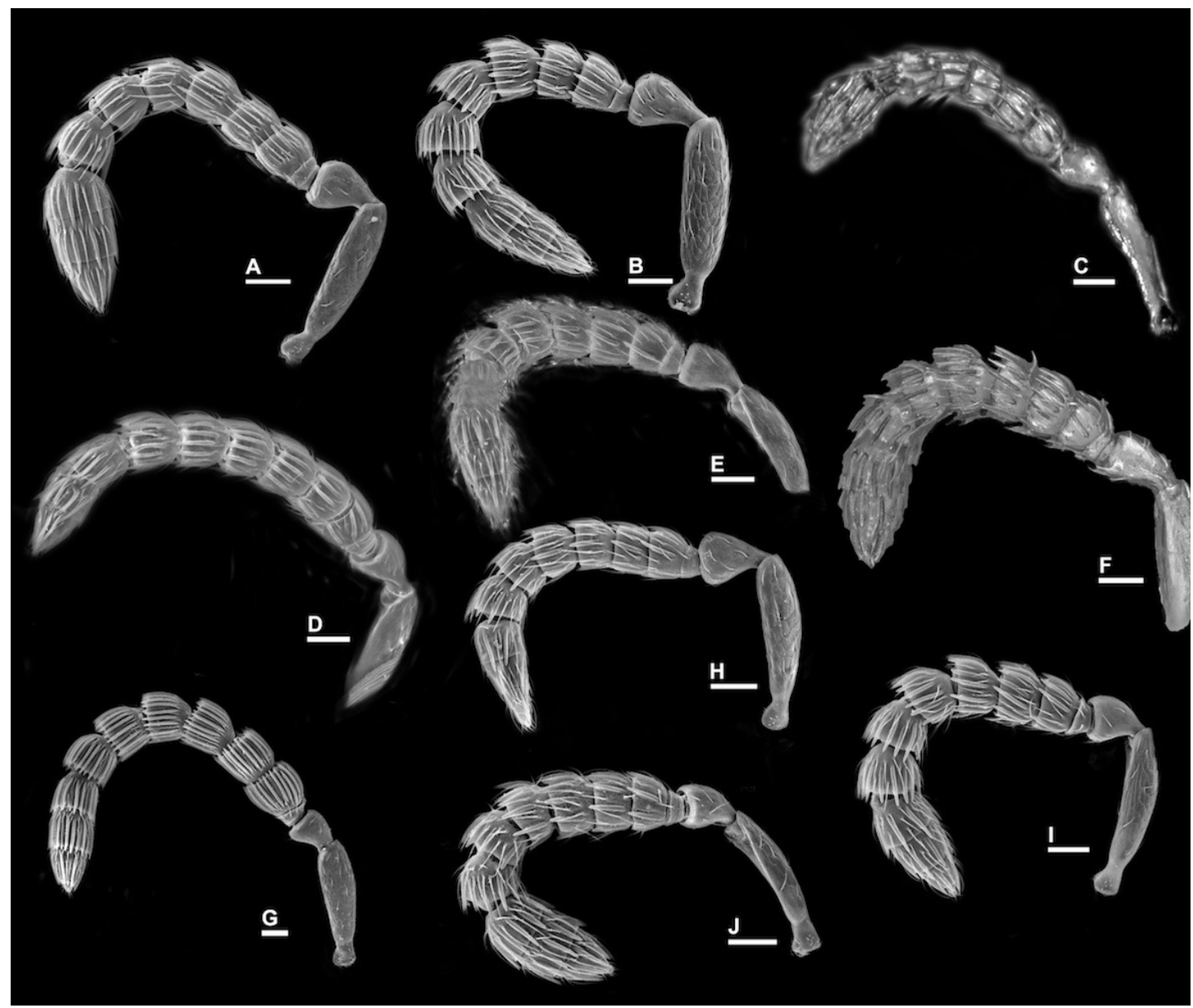

1483

Fig. 7: Antenna, Idarnes incertus sp. group, females. A, I. flavicrus sp. n.; B, I. flaviventris sp. n.;

1485 C, I. gibberosus sp. n.; D, I. gordhi sp. n.; E, I. hansoni Bouček, 1993, Paratype; F, I. aff. 1486 incertus Ashmead, 1900 (JRAS01219), Paralectotype; G, I. maximus sp. n.; H, I. nigriventris sp. 1487 n.; I, I. pseudoflavus sp. n.; J, I. ramirezi sp. n. Scale $=50 \mu \mathrm{m}$. 


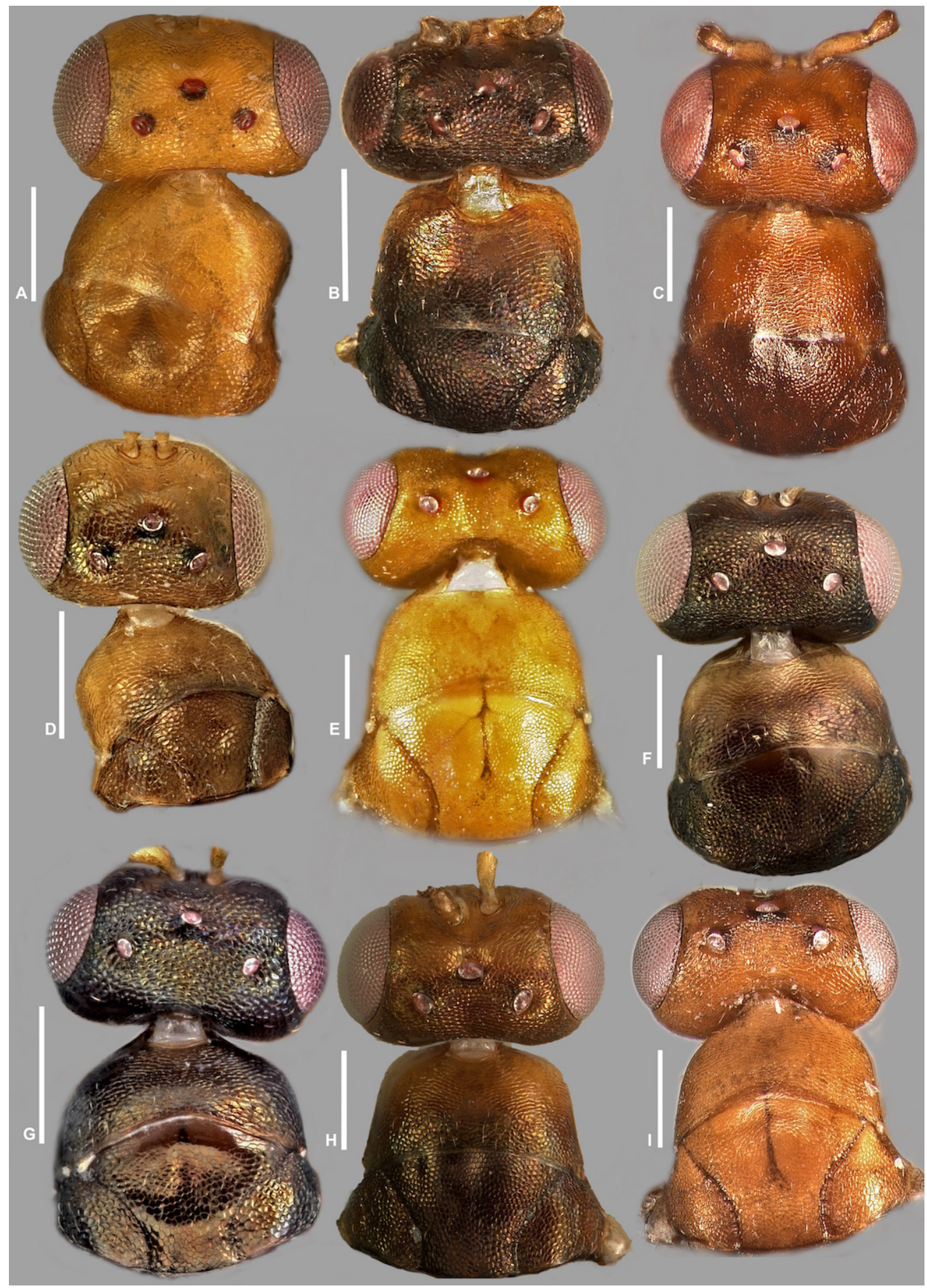

1489 Fig. 8: Head and mesosoma (part), Idarnes incertus sp. group, females. A, I. amacayacuensis sp. 1490 n.; B, I. amazonicus sp. n.; C, I. americanae sp. n.; D, I. badiovertex sp. n.; E, I. brevis sp. n.; F, 
1491 I. brunneus sp. n.; G, I. comptoni sp. n.; H, I. cremersiae sp. n.; I, I. dimorphicus sp. n. Scale = $1492200 \mu \mathrm{m}$.

1493

1494

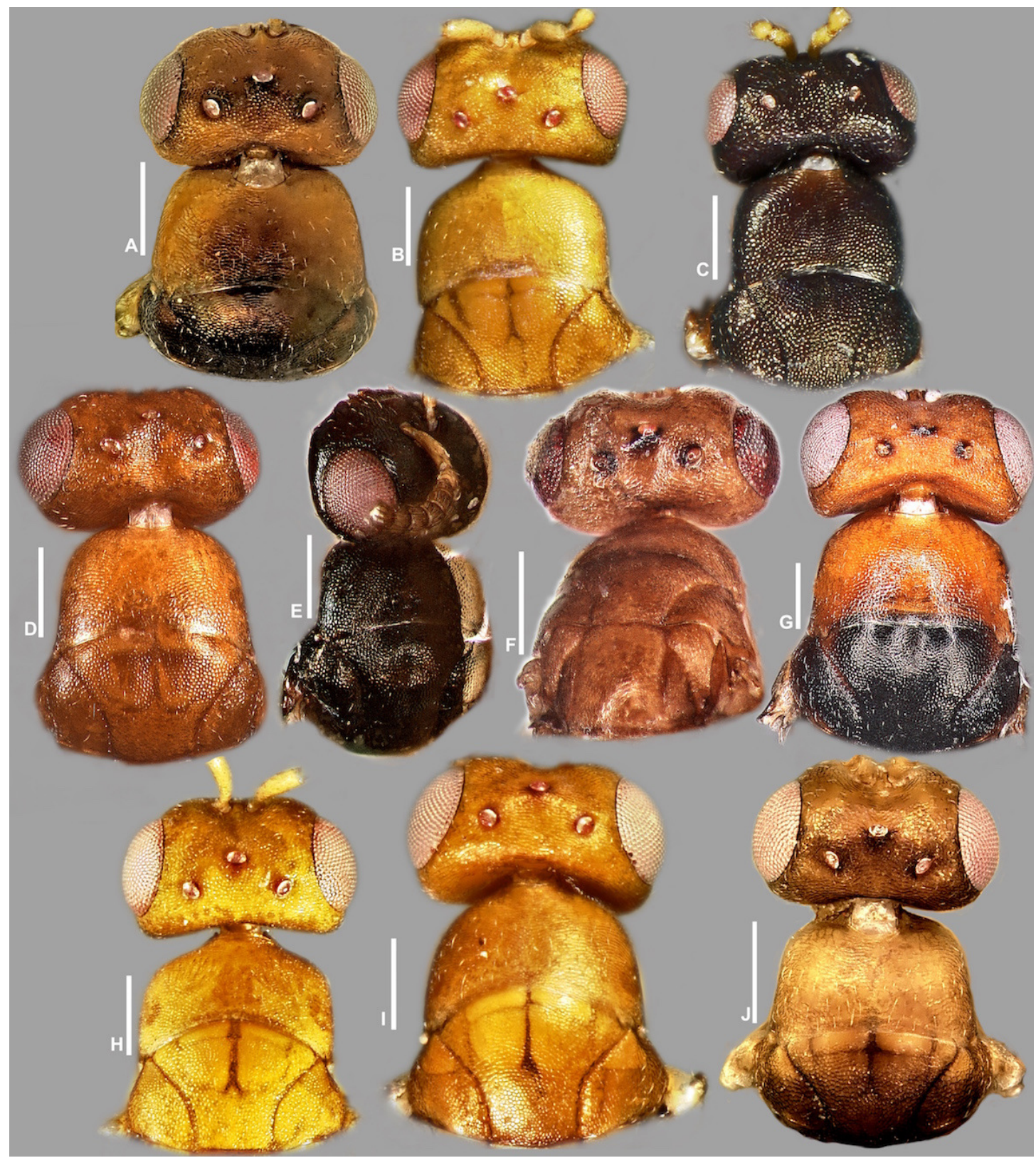

1495 Fig. 9: Head and mesosoma (part), Idarnes incertus sp. group, females. A, I. flavicrus sp. n.; B, I. 1496 flaviventris sp. n.; C, I. gibberosus sp. n.; D, I. gordhi sp. n.; E, I. hansoni Bouček, 1993, 1497 Paratype; F, I. incertus Ashmead, 1900, Paralectotype USNM; G, I. maximus sp. n.; H, I. 1498 nigriventris sp. n.; I, I. pseudoflavus sp. n.; J, I. ramirezi sp. n. Scale $=200 \mu \mathrm{m}$. 


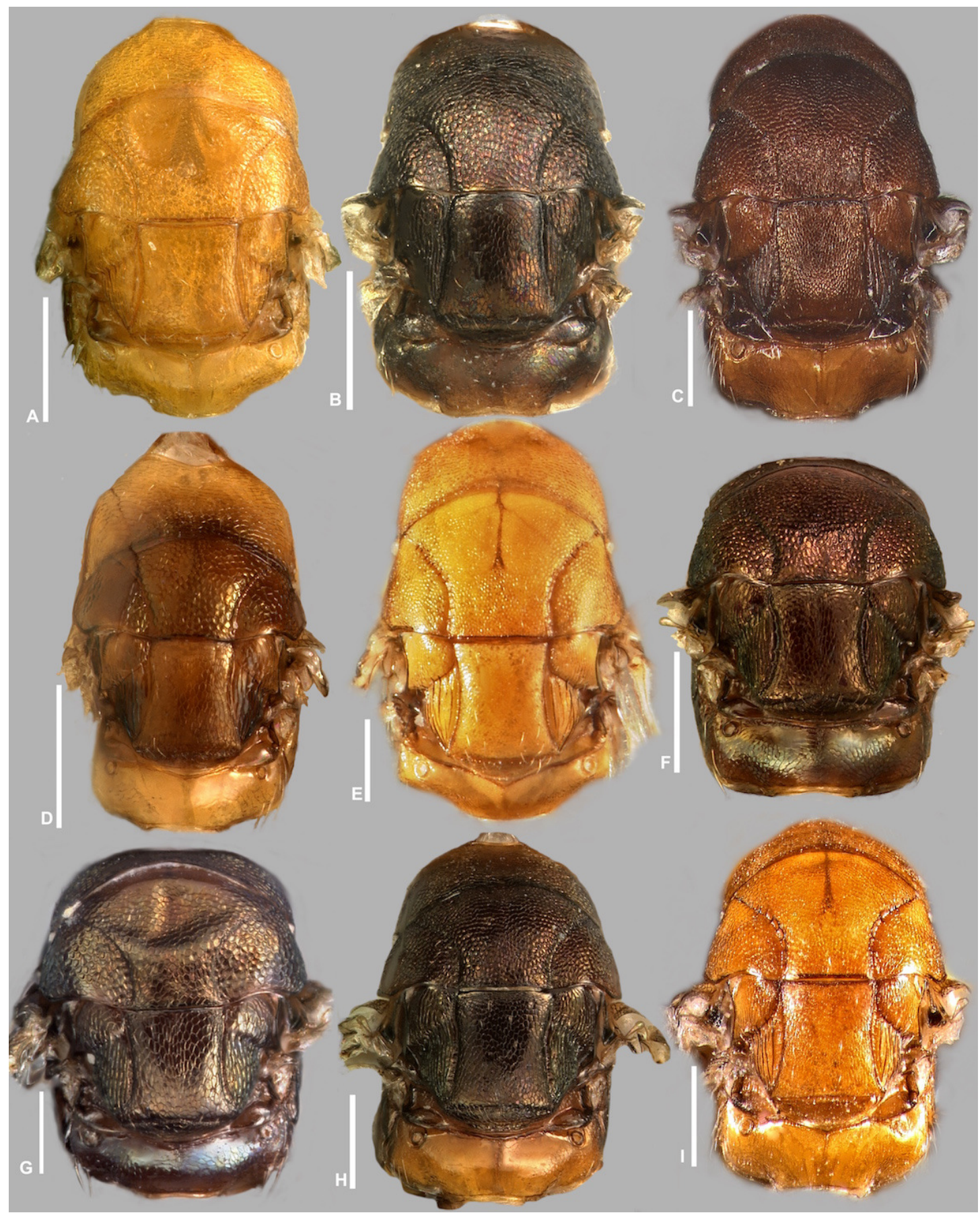

Fig. 10: Mesosoma in dorsal view, Idarnes incertus sp. group, females A, I. amacayacuensis sp.

1501 n.; B, I. amazonicus sp. n.; C, I. americanae sp. n.; D, I. badiovertex sp. n.; E, I. brevis sp. n.; F, 1502 I. brunneus sp. n.; G, I. comptoni sp. n.; H, I. cremersiae sp. n.; I, I. dimorphicus sp. n. Scale = $1503200 \mu \mathrm{m}$. 


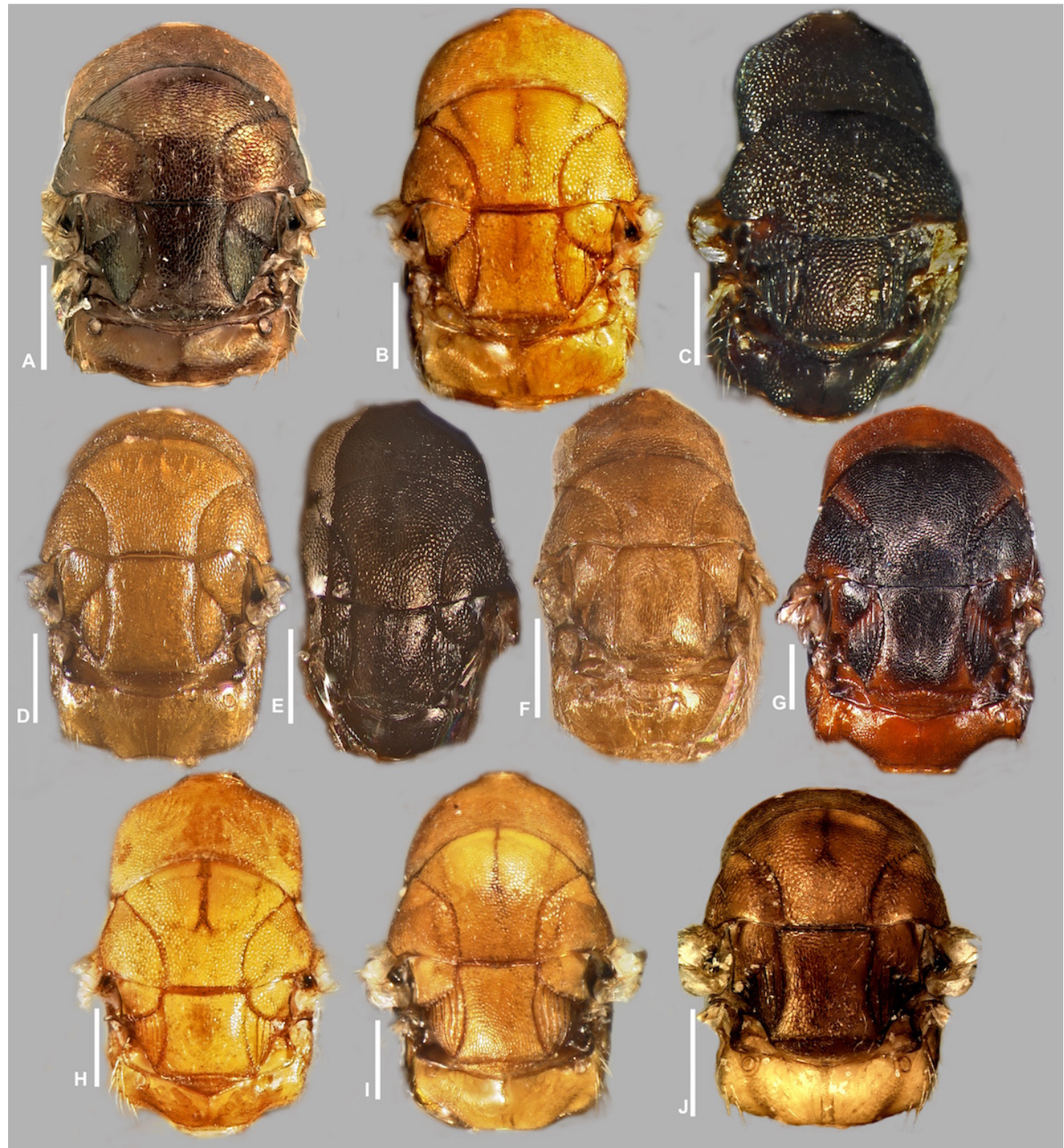

1505 Fig. 11: Mesosoma in dorsal view, Idarnes incertus sp. group, females. A, I. flavicrus sp. n.; B, I. 1506 flaviventris sp. n.; C, I. gibberosus sp. n.; D, I. gordhi sp. n.; E, I. hansoni Bouček, 1993, 1507 Paratype; F, I. incertus Ashmead, 1900, Paralectotype USNM; G, I. maximus sp. n.; H, I. 1508 nigriventris sp. n.; I, I. pseudoflavus sp. n.; J, I. ramirezi sp. n. Scale $=200 \mu \mathrm{m}$. 


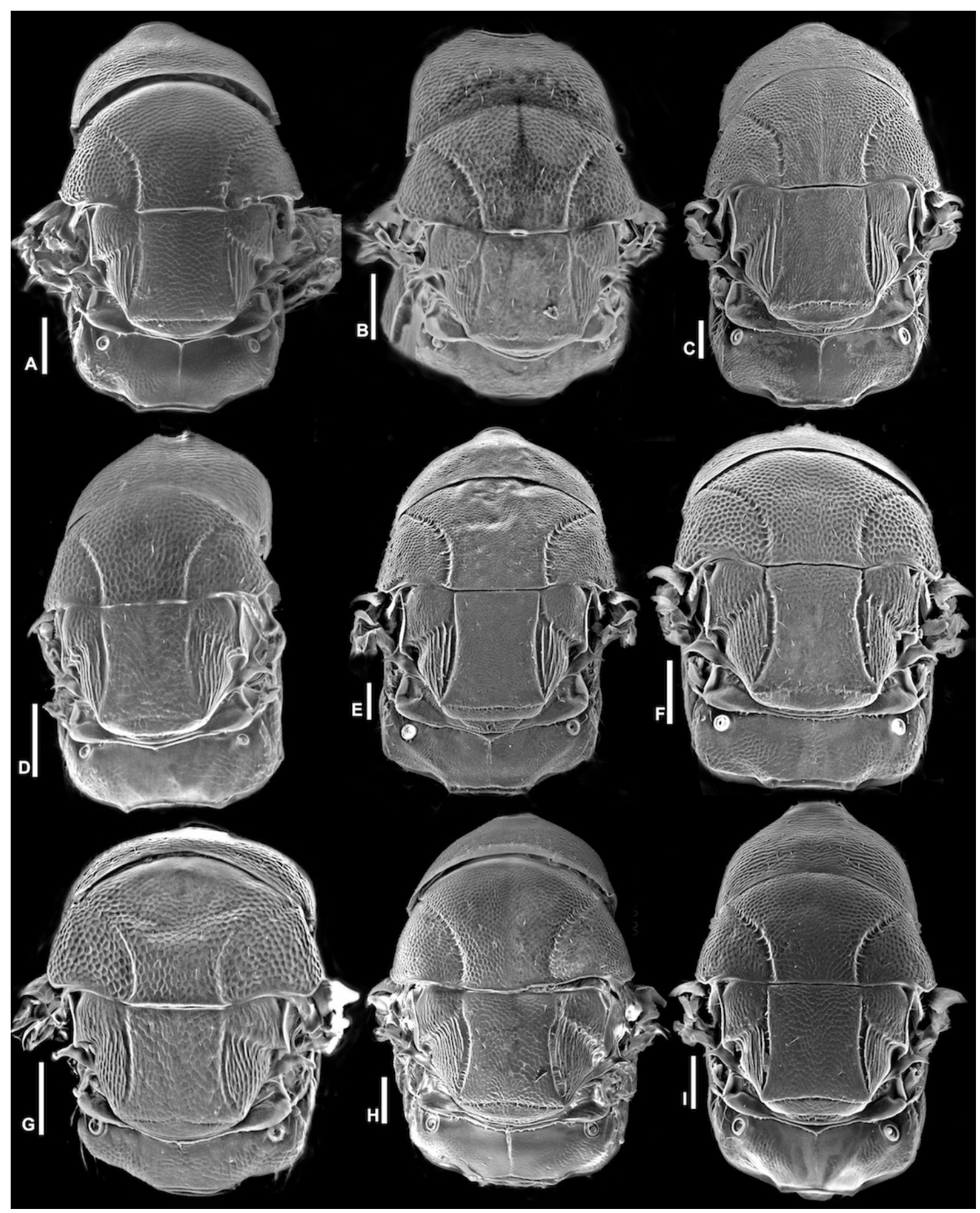

1509

1510 Fig. 12: Mesosoma in dorsal view (SEM), Idarnes incertus sp. group, females A, $I$.

1511 amacayacuensis sp. n.; B, I. amazonicus sp. n.; C, I. americanae sp. n.; D, I. badiovertex sp. n.;

1512 E, I. brevis sp. n.; F, I. brunneus sp. n.; G, I. comptoni sp. n.; H, I. cremersiae sp. n.; I, I.

1513 dimorphicus sp. n. Scale $=100 \mu \mathrm{m}$. 


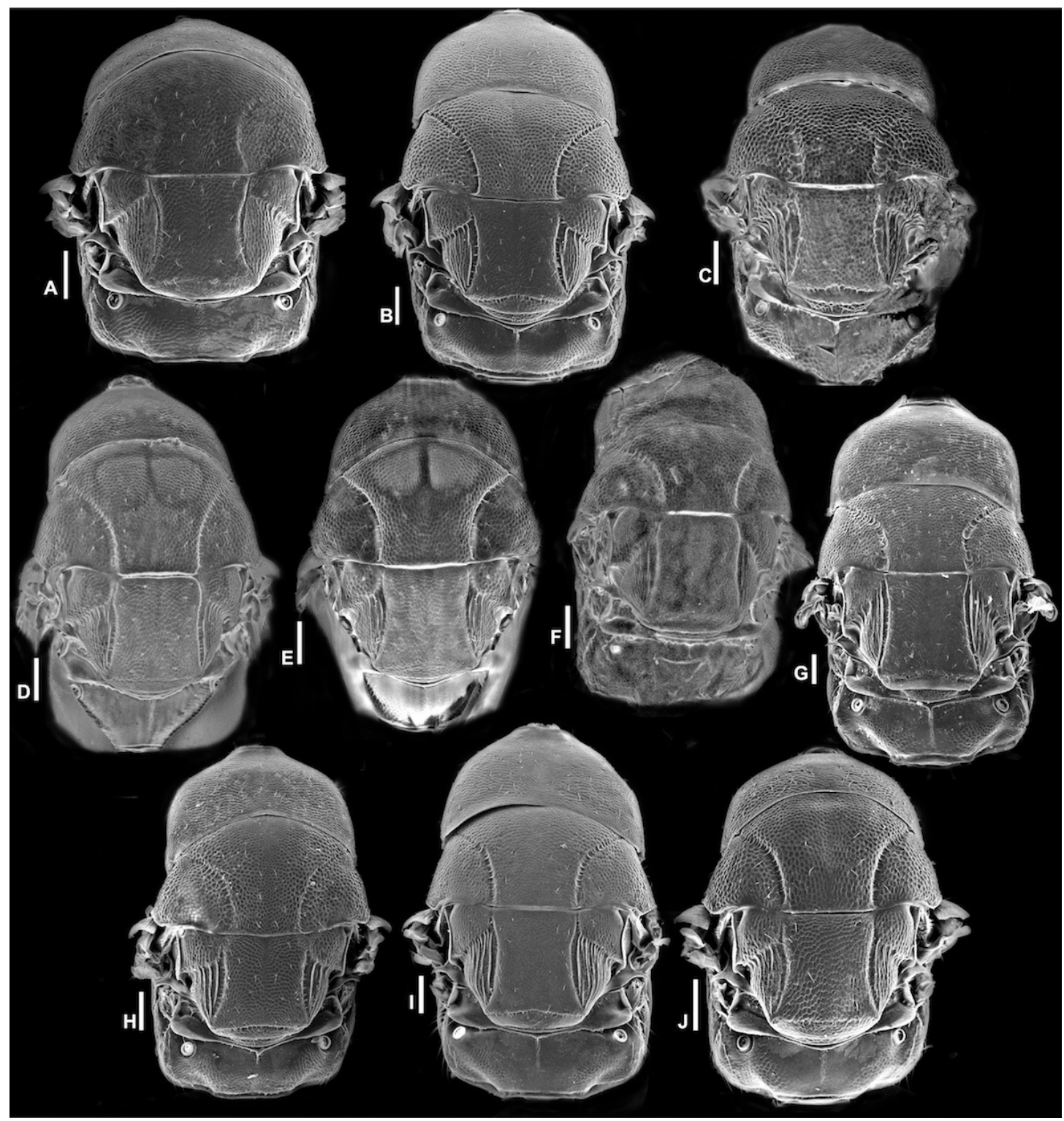

1515 Fig. 13: Mesosoma in dorsal view (SEM), Idarnes incertus sp. group, females. A, I. flavicrus sp.

1516 n.; B, I. flaviventris sp. n.; C, I. gibberosus sp. n.; D, I. gordhi sp. n.; E, I. hansoni Bouček, 1993, 1517 Paratype; F, I. incertus Ashmead, 1900, Paralectotype USNM; G, I. maximus sp. n.; H, I. 1518 nigriventris sp. n.; I, I. pseudoflavus sp. n.; J, I. ramirezi sp. n. Scale $=100 \mu \mathrm{m}$. 


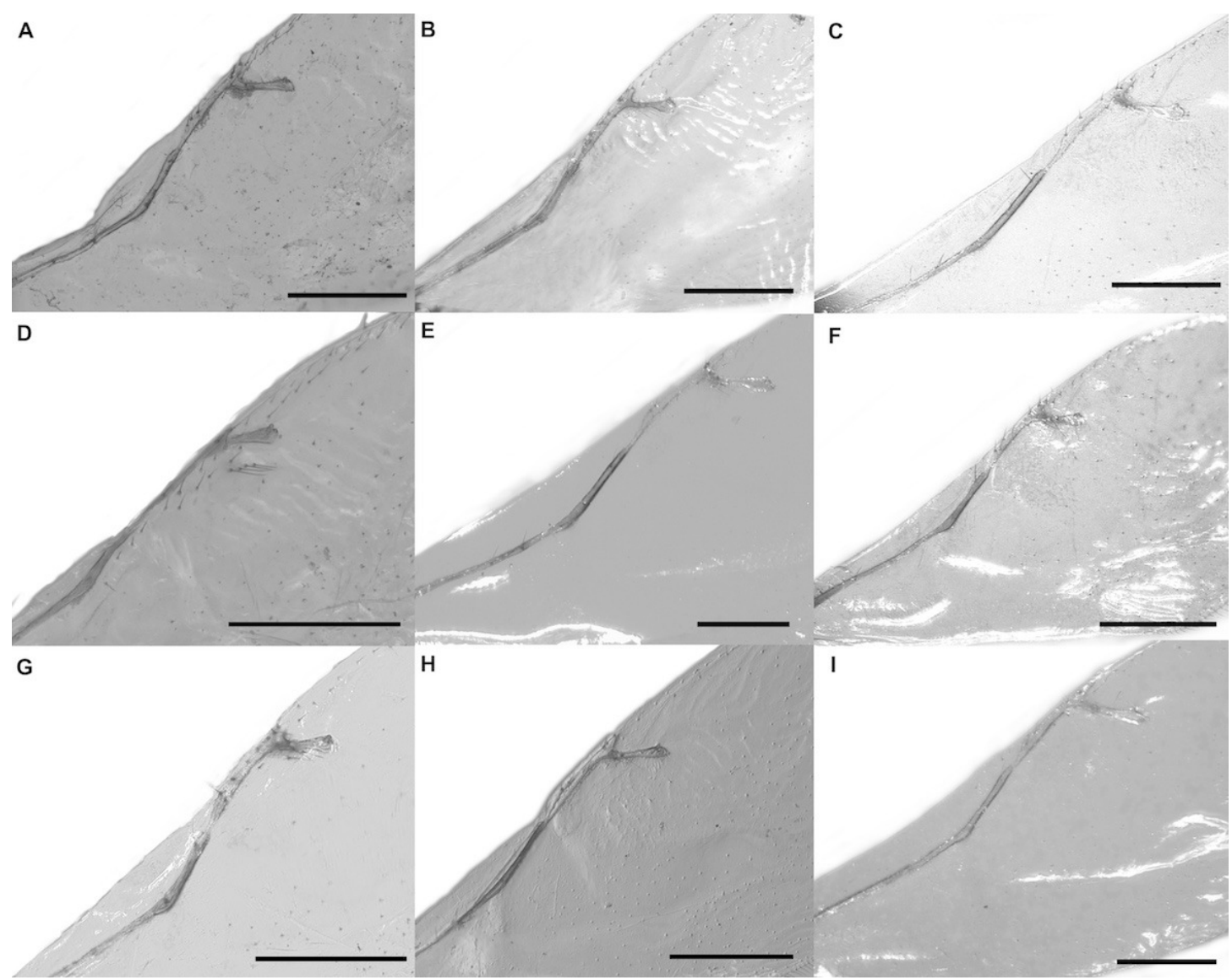

Fig. 14: Wing venation, Idarnes incertus sp. group, females. A, I. amacayacuensis sp. n.; B, I. 1521 amazonicus sp. n.; C, I. americanae sp. n.; D, I. badiovertex sp. n.; E, I. brevis sp. n.; F, I. 1522 brunneus sp. n.; G, I. comptoni sp. n.; H, I. cremersiae sp. n.; I, I. dimorphicus sp. n. Scale $=200$ $1523 \mu \mathrm{m}$. 


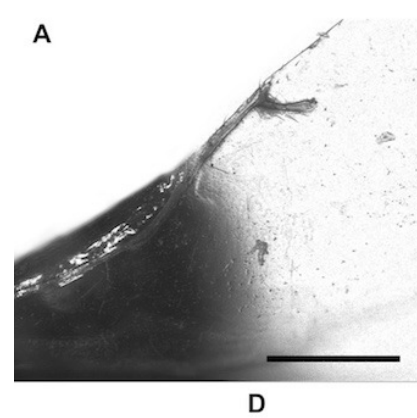

B

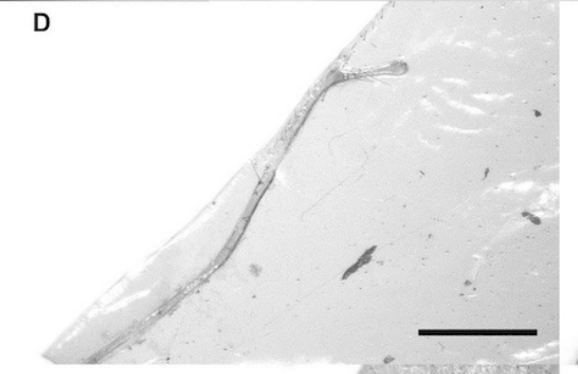

H

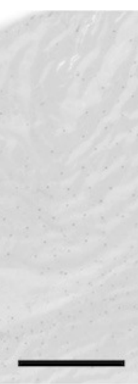

(1)

C
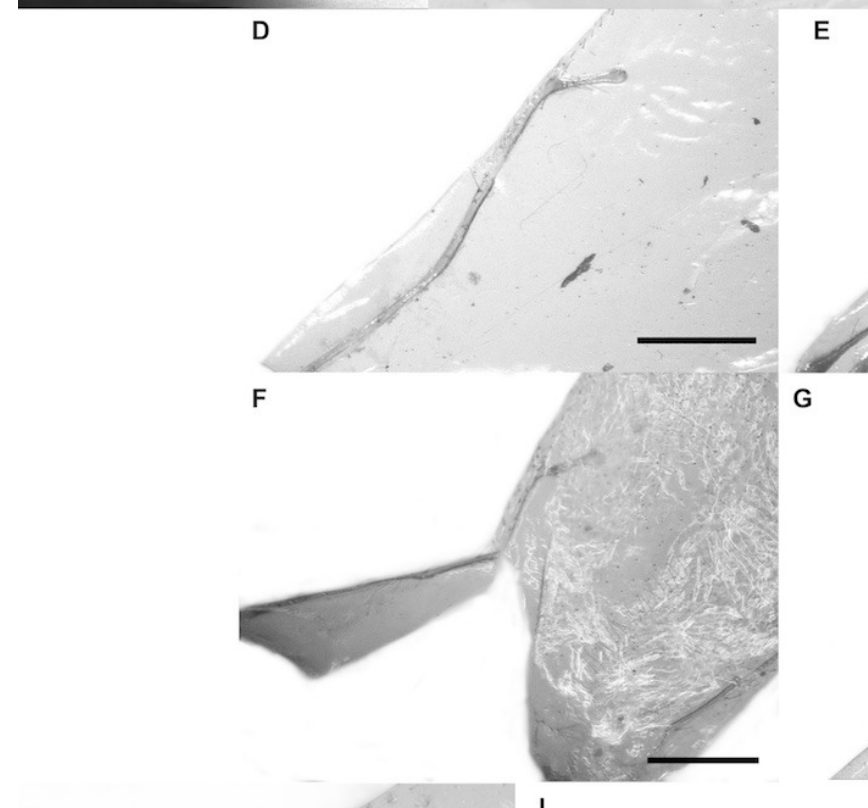

G
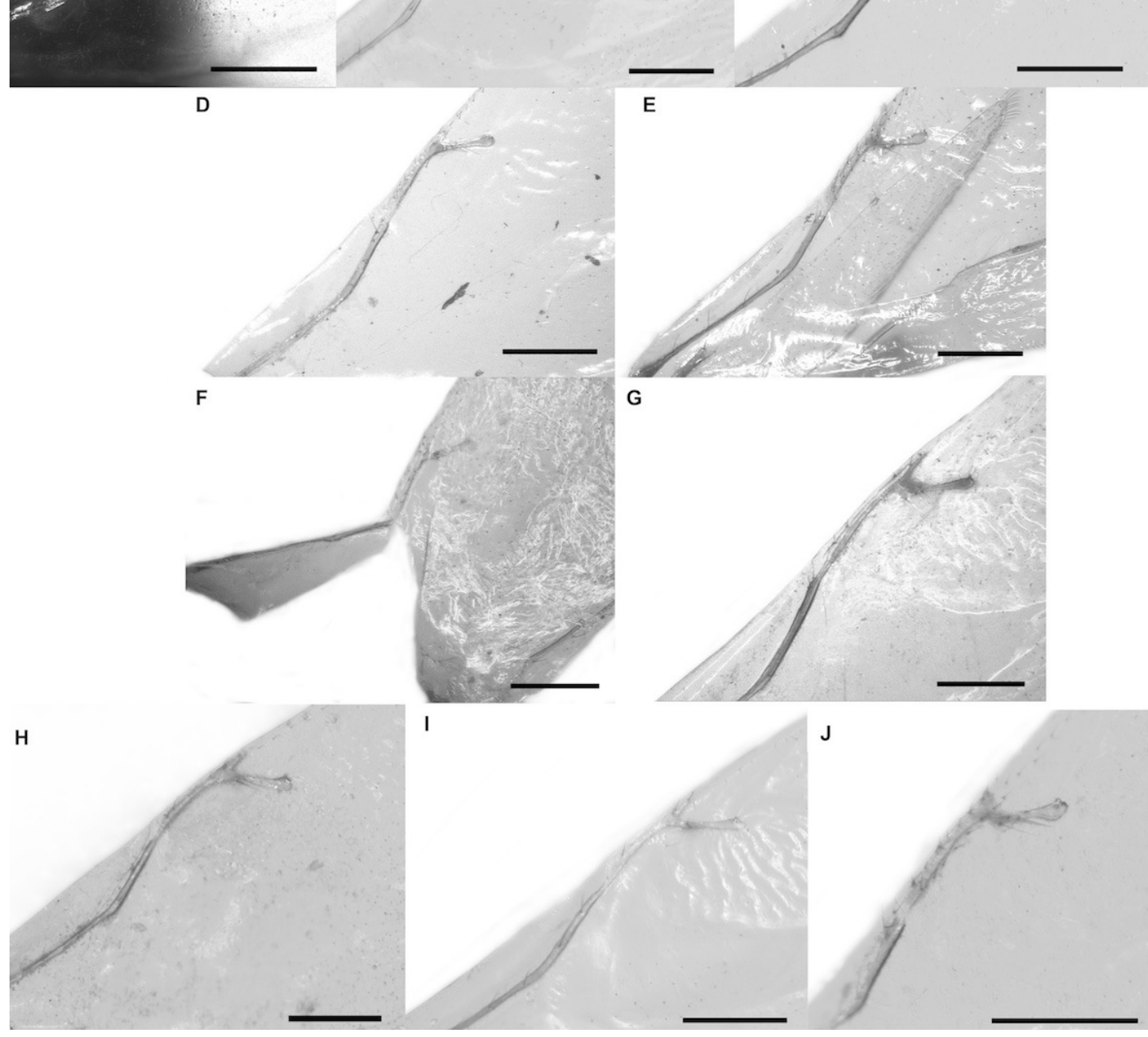

I
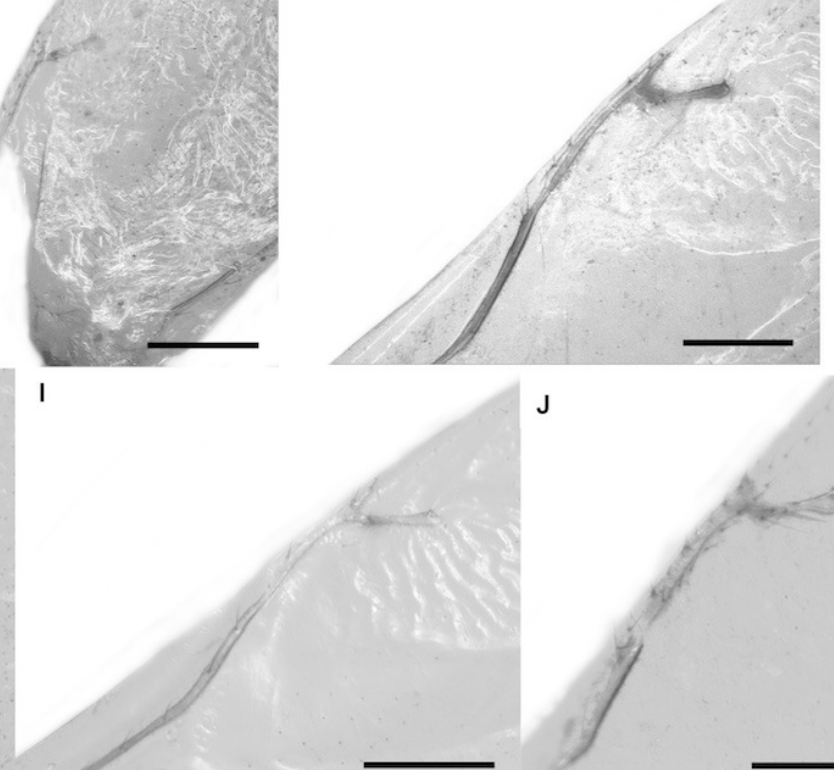

J

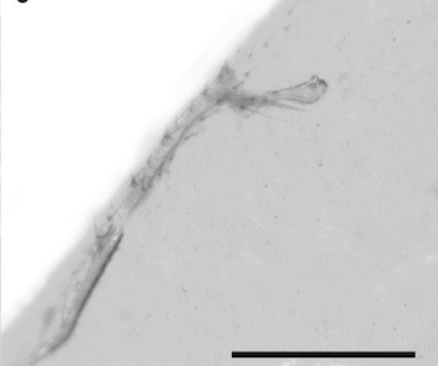

1525 Fig. 15: Wing venation, Idarnes incertus sp. group, females. A, I. flavicrus sp. n.; B, I. 1526 flaviventris sp. n.; C, I. gibberosus sp. n.; D, I. gordhi sp. n.; E, I. hansoni Bouček, 1993, 1527 Paratype; F, I. incertus Ashmead, 1900, Paralectotype USNM; G, I. maximus sp. n.; H, I. 1528 nigriventris sp. n.; I, I. pseudoflavus sp. n.; J, I. ramirezi sp. n. Scale $=200 \mu \mathrm{m}$. 


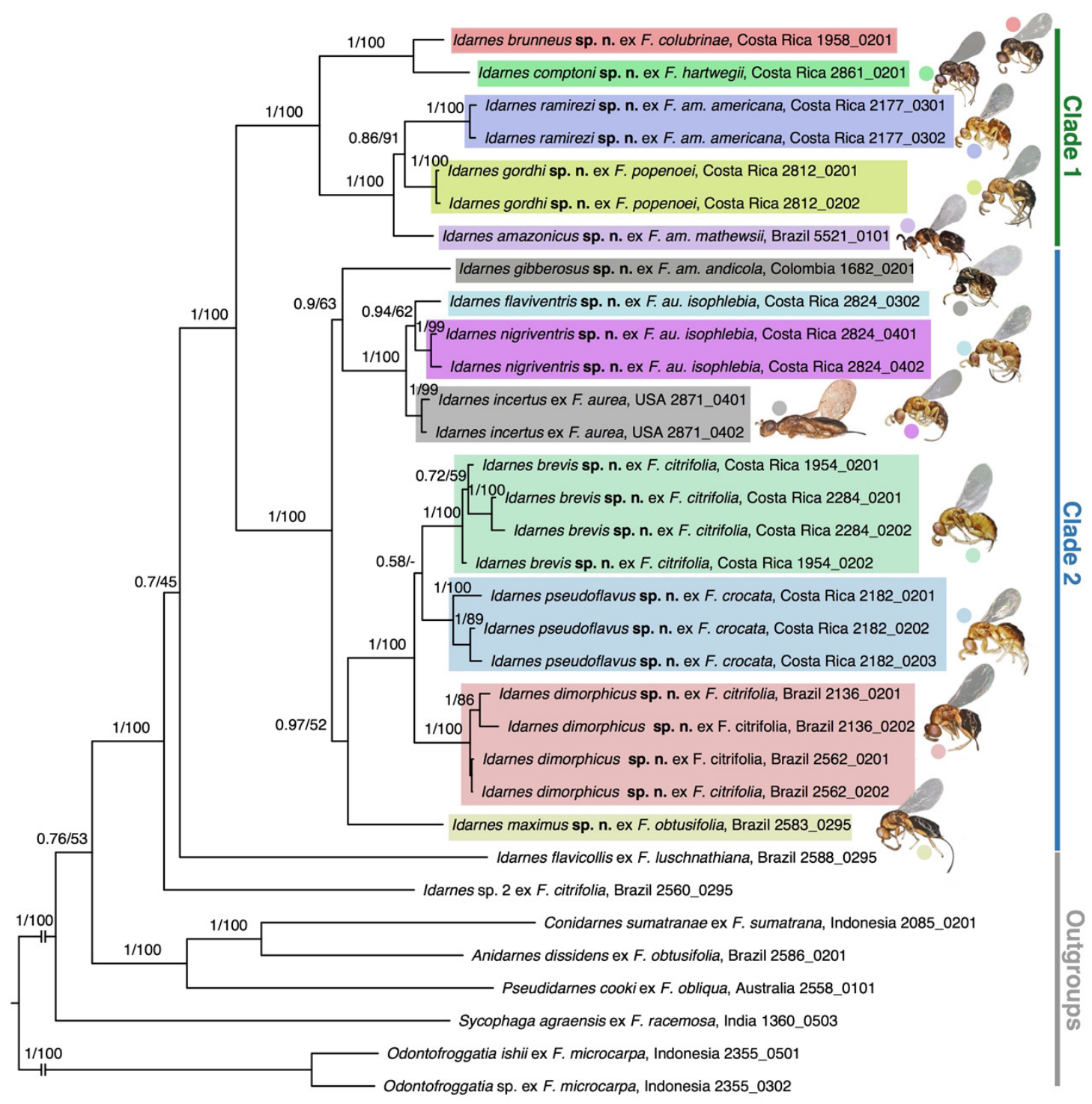

1530 Fig. 16: Phylogram of relationships among the Idarnes incertus species-group species and eight outgroup species obtained with Bayesian inference. Bayesian posterior probabilities (decimals)

1532 and likelihood bootstrap values (percentage) are indicated above nodes (PP/ $\left.\mathrm{ML}_{\mathrm{BP}}\right)$. Boxes

1533 indicate specimens belonging to a same species. F. am. =F. americana; F. au. = F. aurea. 